\title{
Random-Singlet Phase in Disordered Two-Dimensional Quantum Magnets
}

\author{
Lu Liu, ${ }^{1}$ Hui Shao, ${ }^{2,3}$ Yu-Cheng Lin, ${ }^{4, *}$ Wenan Guo, ${ }^{1,2, \dagger}$ and Anders W. Sandvik ${ }^{5,3,2, \$}$ \\ ${ }^{1}$ Department of Physics, Beijing Normal University, Beijing 100875, China \\ ${ }^{2}$ Beijing Computational Science Research Center, Beijing 100193, China \\ ${ }^{3}$ Department of Physics, Boston University, 590 Commonwealth Avenue, Boston, Massachusetts 02215, USA \\ ${ }^{4}$ Graduate Institute of Applied Physics, National Chengchi University, Taipei 11605, Taiwan \\ ${ }^{5}$ Beijing National Laboratory for Condensed Matter Physics and Institute of Physics, \\ Chinese Academy of Sciences, Beijing 100190, China
}

(Received 17 April 2018; revised manuscript received 13 September 2018; published 5 December 2018)

We study the effects of disorder (quenched randomness) in a two-dimensional square-lattice $S=1 / 2$ quantum-spin system, the $J-Q$ model with a multispin interaction $Q$ supplementing the Heisenberg exchange $J$. In the absence of disorder, the system hosts antiferromagnetic (AFM) and columnar valence-bond-solid (VBS) ground states. The VBS breaks $Z_{4}$ symmetry spontaneously, and in the presence of arbitrarily weak disorder it forms domains. Using quantum Monte Carlo simulations, we demonstrate two different kinds of such disordered VBS states. Upon dilution, a removed site in one sublattice forces a leftover localized spin in the opposite sublattice. Such spins interact through the host system and always form AFM order. In the case of random- $J$ or $-Q$ interactions in the intact lattice, we find a different spin-liquid-like state with no magnetic or VBS order but with algebraically decaying mean correlations. Here we identify localized spinons at the nexus of domain walls separating regions with the four different VBS patterns. These spinons form correlated groups with the same number of spinons and antispinons. Within such a group, we argue that there is a strong tendency to singlet formation because of the native pairing and relatively strong spinon-spinon interactions mediated by the domain walls. Thus, the spinon groups are effectively isolated from each other and no long-range AFM order forms. The mean spin correlations decay as $r^{-2}$ as a function of distance $r$. We propose that this state is a two-dimensional analogue of the well-known random-singlet (RS) state in one dimension, though, in contrast to the one-dimensional case the dynamic exponent $z$ is finite in two dimensions. By studying quantum-critical scaling of the magnetic susceptibility, we find that $z$ varies, taking the value $z=2$ at the AFM-RS phase boundary and growing upon moving into the RS phase (thus, causing a power-law divergent susceptibility). The RS state discovered here in a system without geometric frustration may correspond to the same fixed point as the RS state recently proposed for frustrated systems, and the ability to study it without Monte Carlo sign problems opens up opportunities for further detailed characterization of its static and dynamic properties. We also discuss experimental evidence of the RS phase in the quasi-two-dimensional square-lattice randomexchange quantum magnets $\mathrm{Sr}_{2} \mathrm{CuTe}_{1-x} \mathrm{~W}_{x} \mathrm{O}_{6}$ for $x$ in the range of 0.2-0.5.

DOI: 10.1103/PhysRevX.8.041040

\section{INTRODUCTION}

In the quest to classify and characterize ground states and excitations of quantum many-body systems, disorder (quenched randomness) plays a central role. Beyond the fundamental scientific interest in understanding the interplay

\footnotetext{
yc.lin@nccu.edu.tw

†waguo@bnu.edu.cn

*sandvik@bu.edu
}

Published by the American Physical Society under the terms of the Creative Commons Attribution 4.0 International license. Further distribution of this work must maintain attribution to the author(s) and the published article's title, journal citation, and DOI.

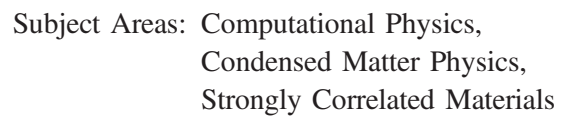

between quantum fluctuations and intrinsic randomness, there are also potential practical implications: In the same way as pure crystalline states of matter are often not optimal for achieving desired properties of materials, e.g., in the case of metals hardened by limiting the size of crystal grains, it is likely that quantum technologies will emerge that exploit disorder effects. For example, random spin chains have been proposed as key elements for memories $[1,2]$ and state transfer channels [3] in quantum computing. Twodimensional (2D) quantum-spin systems, which we consider here, are other natural settings for exploring novel disorderinduced states.

Recent experimental efforts have been devoted to searches for quantum-spin liquids in quasi-2D insulators. Several candidate systems showing the qualitative signatures of spin 
liquids have been identified, e.g., in a series of organic salts where the spins reside on triangular lattices [4-7] and in the kagome-lattice herbertsmithite [8-11]. It has so far not been possible to unambiguously match the properties of these systems to theoretically proposed spin liquids, however, and it has been suggested that disorder effects are crucial for understanding the observed behaviors [12]. In a more extreme interpretation put forward recently [13-20], disorder is even responsible for realizing a certain spin liquid, the random-singlet (RS) state, in some triangular, kagome, and frustrated honeycomb lattice systems, e.g., the triangular $\mathrm{YbMgGaO}_{4}[21,22]$ where disorder is present in the form of random occupation of $\mathrm{Mg}$ and $\mathrm{Ga}$ ions on equivalent lattice sites between the magnetic layers. While such a state has not yet been observed in systems without geometric frustration, there is recent experimental evidence for an RS state in a square-lattice system: the double perovskite $\mathrm{Sr}_{2} \mathrm{CuTe}_{1-x} \mathrm{~W}_{x} \mathrm{O}_{6}$. Here, the disorder is in the form of random $\mathrm{Te} \leftrightarrow \mathrm{W}$ substitutions relative to the isostructural compounds $\mathrm{Sr}_{2} \mathrm{CuTeO}_{6}$ and $\mathrm{Sr}_{2} \mathrm{CuWO}_{6}$, which have dominant nearest- and next-nearest-neighbor spin interactions, respectively [23-25].

Here we show that disorder can induce a spin-liquid-like state - a gapless state with algebraic correlation functionsin a $2 \mathrm{D}$ quantum spin system on the square lattice even without geometric frustration. We refer to this state as an RS state for reasons to be discussed further in Sec. II. To demonstrate the existence of the RS state and to characterize its properties, we carry out large-scale quantum Monte Carlo (QMC) simulations of an $S=1 / 2$ quantum-spin model, the $J-Q$ model, which in the absence of disorder hosts both a Néel antiferromagnetic (AFM) and a spontaneously singlet-dimerized valence-bond solid (VBS) ground state. The transition between these states is driven by enhancing the formation of correlated singlets by increasing the multispin (here six-spin) interaction $Q$, which competes with the Heisenberg exchange $J$. We show that randomness in the coupling constants leads to the formation of domains in the fourfold degenerate VBS state, with different realizations of the bond order and with domain walls of the type expected [26] to lead to localized spinons at each nexus of four domain walls. These spinons form in correlated groups of even numbers, as a consequence of the domain-wall topology. We show evidence for domain-wall-mediated enhanced spinon-spinon interactions, which leads to singlet formation within the groups and no residual AFM ordering of the spinons. As a contrast, we also consider a site-diluted system in which the remnant local moments associated with removed sites are not spatially strongly correlated; thus, residual AFM order forms and there is no RS phase.

We present a broad survey of the phase diagrams, quantum-phase transitions, and basic ground-state and temperature $T>0$ properties of the $2 \mathrm{D}$ RS phase in different versions of the random $J-Q$ model. The spin and bond correlations at $T=0$ decay as power laws, likely as a consequence of rare events in the form of singlet formation over large distances. At $T>0$, using lattices sufficiently large to reach the thermodynamic limit, we find power-law scaling in $T$ of the magnetic susceptibility. This behavior allows us to extract the dynamic exponent $z$, which we find is varying inside the RS phase.

It is possible that the RS state we identify here is the same one, in the sense of renormalization-group (RG) fixed points, as the one proposed recently to arise out of a VBS on the triangular lattice in the presence of random couplings [18]. It may then also be a realization of the unusual magnetic states observed in $\mathrm{YbMgGaO}_{4}$ and $\mathrm{Sr}_{2} \mathrm{CuTe}_{1-x} \mathrm{~W}_{x} \mathrm{O}_{6}$, and possibly in many other disordered spin-liquid candidates as well. The possibility of creating this state with a "designer Hamiltonian" within the $J-Q$ family of models is very significant, as this unfrustrated (in the geometric sense) system is amenable to large-scale QMC studies without the sign problems plaguing simulations of models with frustration. Thus, the RS state in these systems can be characterized essentially completely-far beyond the analytical calculations in Ref. [18] and the exact diagonalization (ED) numerics on small frustrated Heisenberg lattices in Refs. [13-17], and on slightly larger triangular lattices by density-matrix renormalization-group (DMRG) calculations in Ref. [20]. In particular, we are able to reliably study the AFM-RS quantum-phase transition.

The paper is organized as follows: In Sec. II, we discuss the broader context of our work and provide specifics of the models considered. In addition to the main focus on different kinds of disorder in the $J-Q$ model, we also discuss a simpler case as a point of reference: the statically columnar-dimerized Heisenberg model in which localized moments different from the VBS spinons form in the neighborhood of removed sites. In Sec. III, in order to aid in the presentation and interpretation of the extensive QMC results in the later sections, we discuss qualitatively the phenomena and mechanisms that we identify as responsible for the RS state, specifically, the pairing of localized spinons and the role of VBS domain walls in mediating effective magnetic interactions. In Sec. IV, we present the results of ground-state projector QMC calculations of static properties of all the models considered, with the main focus on the order parameters and correlation functions in the RS phase in the cases where this state is attained. We demonstrate the existence of a universal continuous AFM-RS quantum-phase transition. In Sec. V, we discuss the susceptibility results at $T>0$, which allow us to extract the dynamic exponent at the AFM-RS transition and in the RS phase. In Sec. VI, we provide evidence for the mechanism underlying the formation of the RS state: spinon interactions mediated by VBS domain walls. We conclude in Sec. VII with a brief summary and further discussion of our results and their significance in the context of both theory and experiments. We reanalyze the recent susceptibility data for $\mathrm{Sr}_{2} \mathrm{CuTe}_{1-x} \mathrm{~W}_{x} \mathrm{O}_{6}$ with $x$ in the range of $0.2-0.5[25]$ and demonstrate that the divergence 
at low $T$ is slower than $1 / T$, consistent with what we find for the RS phase.

\section{BACKGROUND AND MODELS}

\section{A. Infinite-randomness fixed points and the random-singlet phase}

Theoretically, when randomness is a relevant perturbation under RG transformations, fixed points corresponding to ground-state phases and critical points appear beyond those realized in pure, translationally invariant systems $[27,28]$. In some cases, the RG flow converges to nonzero but finite disorder, e.g., at critical points in many quantumspin glasses [29-32], boson systems with random potentials [33] or random hopping [34], and Heisenberg antiferromagnets [35-37]. However, the randomness can also increase without bounds in the RG flow, leading to an infinite-randomness fixed point (IRFP). This broad class of fixed points has been extensively studied using strongdisorder RG (SDRG) methods in quantum systems in one [38-45] and higher dimensions [46-51] (in addition to many applications in classical statistical physics [52]). The most striking general property of the IRFPs is an infinite dynamic exponent $z$; i.e., the scaling relationship between energy $(\epsilon)$ and length $(l)$ scales is exponential instead of the conventional power-law relation $\epsilon \sim l^{-z}$. Moreover, rare instances of long-distance entangled spins (or particles) lead to different behaviors of the mean and typical correlation functions versus distance $r$, decaying, respectively, as a power law and exponentially.

An important example of an IRFP is the 1D RS phase realized in the $S=1 / 2$ Heisenberg and $X X$ chains with random-exchange couplings [38-41]. Here, the SDRG procedure gives the ground state as a single "frozen" configuration of valence bonds (singlet spin pairs), with a characteristic bond-length distribution. The long-distance entangled spins (long valence bonds) lead to mean spin correlations decaying as $r^{-2}[39,41]$ (while the typical correlations decay exponentially), and the entanglement entropy diverges logarithmically with the system size [42].

IRFPs have been identified also in 2D systems, primarily in transverse-field Ising models [48-50]. However, no convincing case of such a phase or critical point has been reported in 2D quantum magnets with $\mathrm{SU}(2)$ spin-isotropic interactions, such as the standard Heisenberg exchange $[35,36,48]$, as far as we are aware. In the $X X$ model [U(1) symmetry] as well, no such fixed point was found [48], though within a certain approximation in the SDRG method a spurious IRFP fixed point appears [51]. Experimentally, it was argued that an IRFP can be realized at the superconductor-metal transition in Ga thin films [53].

If an RS state exists in 2D systems with $\mathrm{SU}(2)$ spin interactions, which we focus on, it should exhibit algebraically decaying mean correlation functions, as in the $1 \mathrm{D}$ case. We are not aware of any strict definition of an
RS state in 2D, and here we simply use this term for a nonuniform singlet state without any long-range order but with power-law decaying correlation functions. Such a state should roughly correspond to a product of frozen singlet pairs as in the 1D case, perhaps with some other distribution of valence-bond lengths and nontrivial spatial bond correlations.

If the 2D RS state also corresponds to an IRFP, the dynamic exponent should presumably be infinite as well. However, an RS state which has finite $z$ can also in principle exist, although such a state corresponding to a RG fixed point at finite-disorder strength does not exist in random Heisenberg chains. Finite-disorder fixed points have been obtained in SDRG calculations on the 2D Heisenberg model with various types of disorder [35,36,48], but it is not clear whether the SDRG method, by its construction and underlying assumptions, produces the correct fixed point when it does not flow to infinite disorder.

As we mention in the Introduction, Sec. I, there are some experimental indications of 2D disorder-induced spin liquids with finite $z$ in frustrated quantum magnets according to interpretations supported by numerical studies of the $S=1 / 2$ Heisenberg antiferromagnet with random couplings on the triangular and kagome lattices [13-15,20] and also on the honeycomb [16] and square [17] lattice with frustrated interactions. These spin-liquid-like states may very well be realizations of an RS state, as proposed. However, a full characterization of the putative RS ground state and its low-temperature thermodynamic properties (i.e., the form of the asymptotic long-distance correlations and the value of the dynamic exponent) was not possible because of the limited lattice sizes accessible to ED [13-17] and DMRG [20] calculations. The recently developed scenario of the RS state arising out of a VBS on the frustrated triangular lattice [18] contains ingredients-VBS domains and localized spinons- that were not discussed in the context of the numerical works.

Here, we consider a class of $S=1 / 2 \mathrm{~J}-Q$ quantum-spin models on the $2 \mathrm{D}$ square lattice, with no geometric frustration but with interactions leading to weakened AFM order or nonmagnetic VBS states on uniform lattices. In systems with random couplings, the dynamic exponent is finite and varying throughout the RS phase, which is a clear indication of a class of finite-disorder RG fixed points. Our results suggest a mechanism of pairing of localized spinons, which leads to the RS state instead of a weakly ordered AFM state (which had been regarded as the most likely state forming in the random VBS in the absence of frustrated interactions [18]). Importantly, this RS state in an unfrustrated, bipartite system can be induced also in cases where the pure host system is not yet in the VBS state (though not in the standard Heisenberg model with random nearest-neighbor couplings [54]) because local VBS domains are still created in response to the disorder. This observation, along with other considerations, suggests a possible universal scenario that connects 
our square-lattice RS state directly to the abovementioned works on frustrated models with various host states $[13-16,18,20]$. To definitely confirm this universality would require more detailed work on the frustrated systems, however, since the frustrated systems have not been characterized to the extent that we are able to do here for the random $J-Q$ models.

\section{B. Random-singlet state in the $2 \mathrm{D} J-Q$ model}

We study a square-lattice Heisenberg antiferromagnet with nearest-neighbor exchange $J$ augmented with certain multispin interactions of strength $Q$ (the $J-Q$ model). The unadulterated translationally invariant model is defined by the Hamiltonian $[55,56]$

$$
H=-J \sum_{\langle i j\rangle} P_{i j}-Q \sum_{\langle i j k l m n\rangle} P_{i j} P_{k l} P_{m n},
$$

where $P_{i j}$ is the singlet projector for two $S=1 / 2$ spins,

$$
P_{i j}=\frac{1}{4}-\mathbf{S}_{i} \cdot \mathbf{S}_{j}
$$

In the sums in Eq. (1), $\langle i j\rangle$ indicates nearest-neighbor sites, and the index pairs $i j, k l$, and $m n$ in $\langle i j k l m n\rangle$ are neighbors forming a horizontal or vertical column, as illustrated in Fig. 1. The summations are over all pairs and columns so that the Hamiltonian respects all the symmetries of the square lattice, including the $90^{\circ}$ rotation symmetry when $J_{x}=J_{y}=J$ and $Q_{x}=Q_{y}=Q$ as we assume in Eq. (1); we do not consider the more general cases $J_{x} \neq J_{y}$ or $Q_{x} \neq Q_{y}$ here. We introduce various forms of disorder in the model, including site dilution and random- $J$ and $-Q$ couplings drawn from suitable distributions; detailed definitions of the different cases are presented in Sec. IV.

In the uniform system, the $Q$ interactions compete against the exchange terms $J$, disfavoring the strong AFM order present for $Q=0$ (the standard 2D Heisenberg model [57]) by producing correlated local singlets. The interactions are not frustrated in the standard (geometric) sense, however, and the model is amenable to large-scale QMC simulations for all positive values of the ratio $g=Q / J$ (with $J \geq 0$, $Q \geq 0$ being of primary interest) [58]. The ground state is

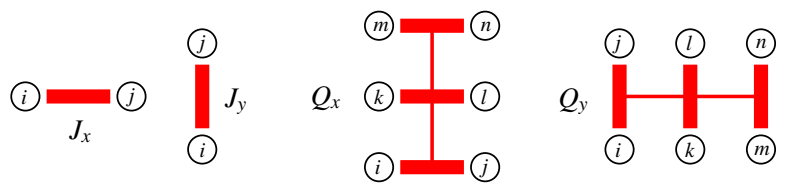

FIG. 1. Illustration of the terms of the $J-Q$ model used in this work. The circles are sites on the square lattice labeled in accordance with the Hamiltonian Eq. (1). The red bars connecting the two sites are the singlet projectors, and the connected bars in the $Q$ terms indicate products. long-range AFM ordered for $g<g_{c}$, with $g_{c} \approx 1.50$ [55] and is a spontaneously dimerized VBS for $g>g_{c}$. In the VBS phase, the $Z_{4}$ symmetry of four degenerate columnar-dimer patterns is broken when $L \rightarrow \infty$.

A columnar VBS state and an AFM-VBS transition is also realized if the $Q$ interaction (often called $Q_{3}$ ) in Eq. (1) is replaced by a simpler one with only two singlet projectors (or $Q_{2}$ ) [59]. The critical coupling ratio $g_{c}$ is then much larger $g_{c} \approx 22$, and the VBS order is rather weak throughout the phase. A much larger number of studies have been devoted to the issue of deconfined quantum criticality within this model [59-66]. Disorder effects on the VBS state are easier to study with the more extended $Q_{3}$ term in Eq. (1), and here we demonstrate RS behavior for a significant range of coupling ratios when either the $J$ or the $Q$ interactions are random. We expect these disorder effects to be generic for VBS phases on bipartite lattices.

Before the advent of the $J-Q$ model, VBS physics was normally associated with geometric frustration in models such as the $J-J^{\prime}$ Heisenberg model with nearest- $(J)$ and next-nearest-neighbor $\left(J^{\prime}\right)$ couplings. These systems are not amenable to large-scale QMC studies because of mixedsign sampling weights (the sign problem), except at the variational level when sampling suitably parametrized and optimized wave functions $[67,68]$. While great progress on frustrated models has been made in the last several years with DMRG and methods based on tensor product states (see, e.g., the recent papers [69-72] for applications to the $J-J^{\prime}$ Heisenberg model), various convergence issues or limited system sizes still make it impossible to carry out calculations as reliable as QMC simulations of sign-problemfree models.

The $J-Q$ models exhibit many of the phenomena of long-standing interest in the context of frustrated quantum magnetism, in particular, the AFM-VBS transition [73], which appears to realize the exotic deconfined quantumcritical (DQC) point scenario [74,75]. It is presently not clear whether exactly this transition is also realized in nonbipartite quantum magnets, e.g., in the square-lattice Heisenberg model with first and second neighbor interactions - there may instead be an extended algebraic spin-liquid phase between the AFM and VBS phases [68-70]. The DQC phenomenon has nevertheless attracted a great deal of interest, as it is a prominent example of a quantum-phase transition beyond the standard LandauGinzburg-Wilson framework. The $J-Q$ models offer unique opportunities to study the emergent degrees of freedom (d.o.f.) - spinons and gauge fields - that are the ingredients of the field-theory description of the DQC point and the VBS phase. A very interesting question is how these d.o.f. respond to quenched disorder; this issue is one aspect of the work presented here.

By the Imry-Ma argument [76], in the presence of even an infinitesimal degree of randomness in the local interactions, the VBS can no longer exist as a long-range 
ordered state due to different columnar-dimerization patterns being energetically favored in different parts of the lattice. Thus, the uniform VBS breaks up into finite domains of different VBS patterns. An extreme case (in the sense of very small VBS domains) of such a disordered dimer state has been dubbed the valence-bond glass [77]. It essentially consists of a random arrangement of short valence bonds and has been discussed in the experimental context of herbertsmithite $[8,9]$ and in certain $3 \mathrm{D}$ frustrated quantum magnets [78,79]. The kagome spin $S=1 / 2$ lattice of herbertsmithite is to some degree diluted with nonmagnetic impurities, and these also liberate spinons from the singlet ground state [12]. It was argued that these spinons interact and form a gapless critical RS state. In this case, the spinons can be regarded as a by-product of the dilution, and in the original picture of the valence-bond glass without dilution [77], there were no such spinons.

In analogy with one-dimensional spin chains with VBS ground states $[41,80]$, and considering the nature of the elementary domain walls in 2D VBS states [26], one should expect a VBS broken up into domains to also have localized spinons at the nexus of domain walls. Therefore, interesting magnetic properties due to local moments can arise even without the explicit introduction of moments by dilution. Indeed, it was very recently argued [18] that a spin-liquidlike state (referred to as an RS state) arises in this way on the triangular lattice when the pristine host system is a VBS. The RS state there is formed as a direct consequence of the randomly interacting localized spinons at the nexus of domain walls. Though spinons do not appear in the scenario discussed in the context of the ED [13-17] and DMRG [20] studies of frustrated systems, localized spinons may still give rise to the physical properties observed in these numerical calculations even though they were not studied explicitly (which would also not be easy with the very small lattices considered). On the square lattice with bipartite interactions, this kind of state has not been previously expected, however, and it was argued that the most likely scenario for systems like the random $J-Q$ model is that the liberated spinons form a subsystem with AFM order instead of a fully disordered RS state [18].

An example illustrated in Fig. 2 of a well-understood system in which residual AFM forms among impurity spins is the diluted columnar-dimerized Heisenberg model, which we later use as a benchmark case for our numerical analysis techniques. For sufficiently large ratio $j_{2}=J_{2} / J_{1}$ of the intra- to interdimer couplings, in the quantumparamagnetic phase, the removed sites leave behind "dangling" spins at the sites near the broken dimers, and these moments form a subsystem with AFM order due to effective bipartite interactions mediated by the inert spingapped dimer host [81]. Thus, the quantum-phase transition out of the AFM ground state at $j_{2} \approx 1.91$ in the intact system $[58,82,83]$ is destroyed and replaced by a crossover from strong to weak AFM order [84,85]. In a disordered

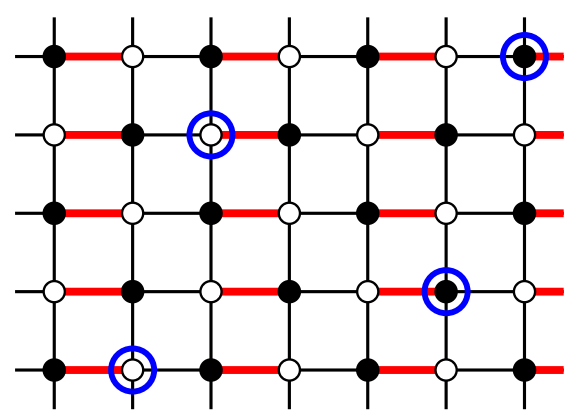

FIG. 2. The statically dimerized $J_{1}-J_{2}$ Heisenberg model with thin black bonds and thick red bonds representing exchange couplings $\mathbf{S}_{i} \cdot \mathbf{S}_{j}$ of strength $J_{1}$ and $J_{2}$, respectively, between $S=$ $1 / 2$ spins. The $A$ and $B$ sublattices are indicated with solid and open black circles. The larger blue circles indicate randomly removed sites. For the intact system with $j_{2}=J_{2} / J_{1}$ larger than $j_{2 c} \approx 1.91[58,82,83]$, the ground state is approximately a product of singlets on the strong bonds, and upon dilution, the "dangling spins" remaining at the "broken dimer" adjacent to each removed spin constitute localized magnetic $S=1 / 2$ moments.

VBS on the square lattice, one might imagine that the disorder-induced spinons should be subject to a similar ordering mechanism [18]. However, our results and arguments suggest that the correlated nature of spinon-antispinon pairs (and larger complexes of even numbers of spinons) was not taken fully into account previously. In particular, we argue that a key missing ingredient in the analysis of bipartite systems (Kimchi et al. [18]) is that the VBS domain walls act as channels of enhanced spinon-spinon interactions within the groups of even numbers of spinons, thus leading to stronger than expected tendency to local singlet formation and, apparently, no residual AFM ordering.

The RS state proposed on the triangular lattice may eventually be unstable to the formation of a quantum-spin glass (a state characterized by randomly frozen moments instead of singlets) according to the arguments by Kimchi et al. [18]. RS physics could then still appear on long length scales and be experimentally relevant, although the asymptotic properties of the system, e.g., the thermodynamics at very low temperatures, would be different. This kind of crossover, with distinct RS behavior up to some length scale or down to some energy scale, may also be expected in the event that the bipartite RS would be unstable to AFM ordering. Here, we find $T=0 \mathrm{RS}$ physics in the random $J-Q$ model and no signs of crossover into weak AFM order up to the largest lattices studied, $64 \times 64$ sites. We also find nontrivial $T>0$ properties that we associate with the RS state. Moreover, we find a distinct transition point with universal critical exponents separating the RS and AFM states. Thus, the RS state appears to be stable.

Though it is not immediately clear whether the RS phase that we identify and characterize here corresponds to the same fixed point as the state identified on the triangular lattice by Kimchi et al. [18], this would be the simplest scenario. We show here that the RS state can also form in 
some cases even though the bipartite host system is not yet VBS ordered but still in the AFM state, as long as there are sufficient interactions (here, $Q$ terms) favoring the formation of some local VBS domains. This role should also be played by standard frustrated interactions in systems with VBS states as well as other states, such as spin liquids or weakly ordered AFMs. The RS state in the disordered $J$ - $Q$ model can then indeed correspond to the same RG fixed point as the states discussed previously in the context of a variety of frustrated host systems, including ED studies [13-17] and DMRG calculations [20]. In the numerical works, the physical picture presented for the nature of the RS state was different, however, with an emphasis in Refs. [13-17] put on the singlet pairs (Anderson localization of singlets) [15] and no reference to the localized spinons and VBS domains emphasized in our work here and in Ref. [18].

In Secs. IV and V, we present $T=0$ and $T>0$ QMC results for the Hamiltonian Eq. (1) with random- $J$ and random- $Q$ couplings, as well as for a site-diluted system with no randomness in the remaining $J$ and $Q$ interactions. For reference, we also present results for the diluted $J_{1}-J_{2}$ Heisenberg model in Fig. 2. To characterize the ground states of these systems in an unbiased way, we use a ground-state projector QMC method formulated in the valence-bond basis [86,87], and to obtain properties at $T>0$, we use the stochastic series expansion (SSE) QMC method [88]. To make the results sections more accessible and concise, in Sec. III we first outline the physical scenario that arises out of the many different calculations reported in the subsequent sections.

\section{DOMAIN WALLS AND SPINONS IN THE DISORDERED VALENCE-BOND SOLID}

On the 2D square lattice and with the bipartite nature of a model such as the $J-Q$ model, the main question regarding the disordered VBS state is whether the spinons localizing at each nexus of four domain walls [26] will form longrange AFM order or some other collective state with only short-range or algebraic spin-spin correlations. As already discussed in Sec. II B, one might suspect [18] that AFM order should exist for all values of $g=Q / J$, in analogy with the fate of the quantum paramagnet and Néelparamagnetic quantum-phase transition in Heisenberg models with static dimerization when spins are randomly diluted (Fig. 2). This picture neglects important spatial correlations among the localized spinons, however, as well as the nature of the VBS domain walls that connect the spinons.

\section{A. Bound spinons as excitations of the pure VBS}

To understand the spatial spinon correlations, consider first an individual, localized spinon created by a topological defect in the VBS (in a pure or random system). As illustrated in Fig. 3, the four lattice bonds pointing out from (a)
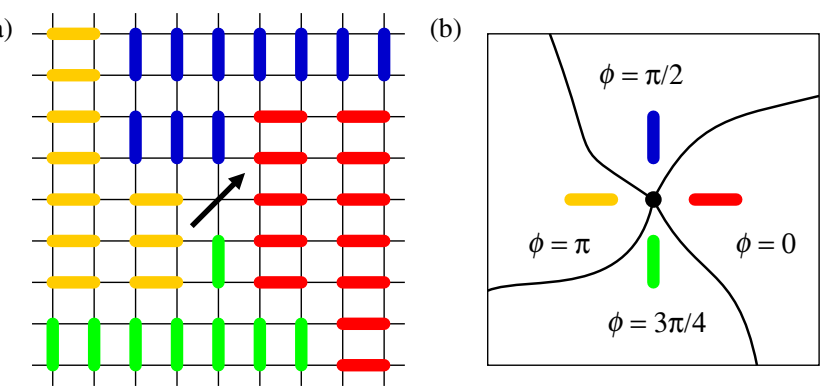

FIG. 3. (a) Illustration of a spinon forming at an unpaired spin at the nexus of domain walls separating the four different columnar VBS patterns on the square lattice. (b) The four VBS patterns associated with angles $\phi$ in a simplified view of the VBS vortex, with only the bonds closest to the core shown in the same color coding as in (a). A similar vortex can also be constructed starting from core bonds rotated by $90^{\circ}$ relative to those shown here.

the site of an unpaired spin (the core of the spinon) correspond to the four different VBS patterns. Another bond arrangement at the unpaired spin can also form, with the bonds rotated by $90^{\circ}$ relative to those in the figure [26], but our simulations of the $J-Q$ model often show the "star" configuration at the spinon (but this local arrangement should not change the properties of the domain walls discussed in Ref. [26]). The four bonds and the corresponding extended VBS domains can be associated with angles $\phi$ as indicated. Note that the energetically favored domain walls correspond to a $\pi / 2$ phase twist [26], while walls with $\pi$ phase change are unstable and break up into two $\pi / 2$ walls (as shown explicitly in Ref. [89]). The $\pi / 2$ domain wall is the origin of the classification of the symmetry of the VBS as the cyclic $Z_{4}$ group, or "clock" symmetry [26,74]. Within a domain wall, the angle $\phi$ (properly defined by coarse graining and averaging over fluctuations) changes continuously, and it is clear that this kind of defect is a vortexlike topological defect of the VBS. Such a vortex forming around a vacancy has been studied with the $J-Q$ model and a field-theoretical description [90]. A spinon should be considered as a composite object of the VBS vortex with the unpaired spin at its core.

Note that a spinon can be associated with either sublattice $A$ or $B$, and the way in which the angle $\phi$ changes, increasing or decreasing, when going around the spinon in a given direction depends on the sublattice. Thus, we can also refer to the two cases as vortices (sublattice $A$ ) or antivortices (sublattice $B$ ), or spinon and antispinon. This classification remains valid also in the presence of longer valence bonds, as long as only bonds connecting the two sublattices are allowed. This restriction is applicable with bipartite interactions, where bonds connecting sites on the same sublattice are always eliminated when a state written in the valence-bond basis is time evolved [91]. Note that fluctuations of the VBS vortices involving longer bonds also lead to the unpaired spin fluctuating around the vortex, 


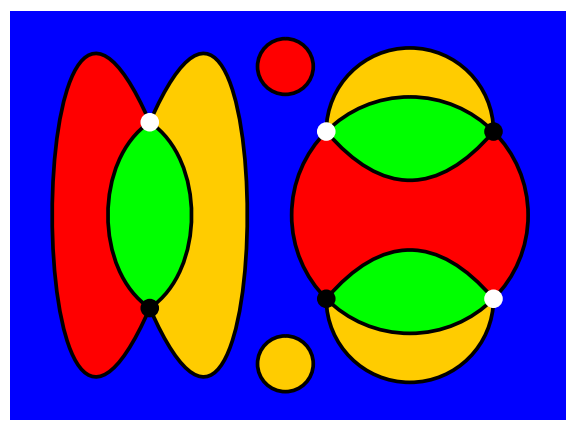

FIG. 4. Illustration of multispinon complexes: a spinon pair (left) with the spinon and antispinon marked as black and white circles, respectively, and a quadruplet (right) consisting of two spinons and two antispinons. Two trivial domains, the yellow and red circles, are also shown. The color coding of the VBS domains is as in Fig. 3 and all domain walls are of the elementary type where the VBS angle twists by $\Delta \phi=\pi / 2$.

instead of being completely localized at the center of the vortex core (and of course the core itself becomes a more extended object).

When exciting the uniform VBS singlet ground state into its low-lying $S=1$ states, spinons always have to be introduced as pairs of spinons and antispinons, and these remain bound to each other as dispersing gapped "triplons." In a simplified static picture, when separating the two members of a triplon, domains form such that each spinon is connected to all four types of domains as in Fig. 3. As shown in Fig. 4, this arrangement leads to a four-stranded confining string, akin to the (more complicated) quarkconfining strings in quantum chromodynamics [92]. Here we do not show the details of the bonds within the domains, only the colors corresponding to the coding in Fig. 3. As already mentioned, in principle, there will also be valence bonds of length greater than one lattice spacing, but the pictures remain valid as long as the probability of longer bonds decays sufficiently rapidly with the bond length. If we consider the total-spin singlet state of the two spinons (an $S=0$ excitation of the VBS), there will also be a bond connecting the spinon and the antispinon sites. Such a long bond corresponds to a small gap between the singlet and triplet excitations (vanishing in the limit of large separation). In the nonrandom VBS, the spinons cannot actually be far separated in this way because other spinons can be excited from the VBS ground state as the string energy becomes sufficiently high. The confining string will then break, thus, limiting the number of bound spinon-antispinon states, again analogous to the case of quark confinement (mesons).

\section{B. Localized spinons in the disordered VBS}

In a system with random couplings, different VBS angles $\phi \in\{0, \pi / 2, \pi, 3 \pi / 2\}$ will be favored in different parts of the system, and the domain size will be governed by the competition of the energy cost of the domain walls and the energy gains due to the disorder. In classical systems, according to the Imry-Ma argument [76], energy minimization always leads to domain formation at $T=0$ in dimensionality $D<2$, while for $D>2$, the uniform state is stable in the presence of weak disorder. Considering entropy effects, the uniform state is also unstable at $T>0$ in $D=2$. Similarly, one can expect quantum fluctuations to also always lead to domain formation in systems with two spatial dimensions at $T=0$ [18]. At least for weak disorder, the domain walls should still be of the $\pi / 2$-twist type. These domain walls can meet in various ways without breaking bonds, but the case of a nexus of four different domains is special and requires the breaking of bonds into unpaired spinons, as in Fig. 3.

As in the uniform VBS state discussed above, spinons forming in a VBS broken up into domains must also always appear in groups of an even number-half of them spinons and half of them antispinons. In Fig. 4, a quadruplet is also shown along with the spinon pair already discussed. It is this inherent correlation among spinons and, importantly, the tendency to singlet formation within the groups, that we believe prohibit the formation of AFM order in the random VBS arising out of the columnar VBS in the $J-Q$ model. The effective interactions between the spinons should be mediated through the domain walls (and we show explicit evidence for this) because they have much smaller local mass gaps than the bulk of the VBS domains (through which interactions between different spinon groups have to be mediated). We also later comment on this picture in the context of SDRG theory.

\section{Basic properties of the RS state}

According to our findings reported in Sec. IV, the abovedescribed disordered VBS state in the $J-Q$ model with random couplings (either random $J$ or random $Q$, both of which we study, or both random, which we do not consider) should be classified as an RS state, a nonuniform spin liquid with mean spin correlations decaying with distance as $r^{-2}$. The form of the spin correlation function is thus the same as in the 1D RS phase, and the dimer (bond singlet) correlations decay with a higher power, likely $r^{-4}$, which again would be the same as in 1D [41]. Unlike the 1D RS state, we do not find a divergent dynamic exponent, however. By investigating the temperature dependence of the uniform magnetic susceptibility, we find $z=2$ ( $T$-independent susceptibility) at the AFM-RS phase boundary and $z>2$ (power-law divergent susceptibility) inside the RS phase.

In further support of a disordered VBS state with no AFM order, we also compare the model with random couplings with a site-diluted $J-Q$ model. Here, like in the diluted $J_{1}-J_{2}$ model in Fig. 2, there will be effective moments associated with the removed sites. Thus, while there may also be localized pair-correlated spinons associated with the meeting points of four domain walls, now there are also moments 


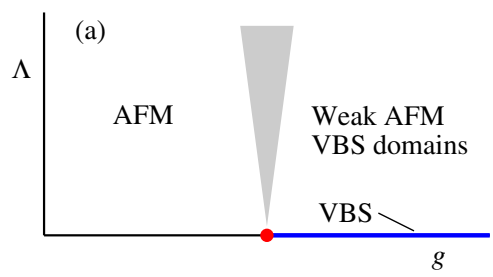

(b)

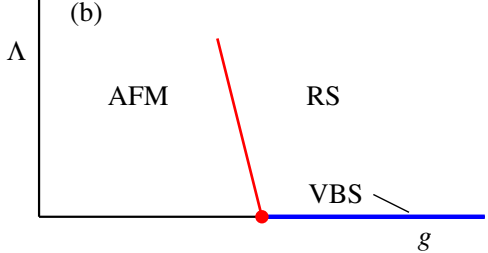

FIG. 5. Schematic ground-state phase diagrams of models such as the $J$ - $Q$ model in the presence of disorder. Here, $\Lambda$ represents a disorder strength, and $g$ is a tuning parameter existing also in the pure system (e.g., $g=Q / J$ in the $J-Q$ model). In the pure model, $\Lambda=0$, there is a DQC point (red circles) separating the AFM and VBS phases. The VBS is destroyed, breaking up into domains for any $\Lambda>0$. In (a), which applies to the model with site dilution, there is no phase transition vs $g$ when $\Lambda>0$, only a crossover (indicated by the wedge) between the standard AFM state and a state with finite VBS domains in which weak AFM order forms among localized effective moments. In (b), which applies to the case of random coupling constants, there is a true continuous quantum-phase transition between the AFM and RS phases for at least some range of $\Lambda>0$.

at random locations without any intrinsic pairing of $A$ and $B$ sublattice moments. The vacancies should also lead to topological defects similar to those discussed above, but, since there is no constraint on their sublattice occupation, it will typically not be possible to pair all the released moments up into spinon-antispinon singlets. The picture of weakly interacting singlet pairs leading to the RS state is then inapplicable. Indeed, in this case we find a VBS broken up into domains and weak AFM order, and no RS state exists in the ground-state phase diagram.

In Fig. 5, we sketch the generic phase diagrams expected based on our findings for the $J-Q$ model in the presence of the different types of disorder discussed in this paper. Here we use a disorder strength denoted by $\Lambda$ on the vertical axis and outlined phases and phase boundaries in the plane $(g, \Lambda)$, where $g$ is the parameter driving the AFM-VBS transition in the clean system $(\Lambda=0)$. In our actual calculations, we vary $g=Q / J$ and study several examples of disorder in $J$ or $Q$, but we do not draw full phase diagrams. we merely detect the relevant phase transitions and study the properties of the phases in certain cases to demonstrate their existence. We expect the phase diagrams in Fig. 5 to be generic for disordered 2D quantum magnets that host AFM-VBS quantum-phase transitions in the absence of disorder.

Note the way the AFM-RS phase boundary is drawn in Fig. 5 as tilted into the AFM phase; i.e., one can reach the RS state not only from the VBS phase of the pure system but also (for some types of disorder) from the AFM state even when it is quite far from the AFM-VBS transition. This destruction of the AFM state can be understood as a tendency to local VBS domain formation in the presence of disorder. On the square lattice, the Heisenberg model with only nearest-neighbor couplings $J$, disorder in the form of random unfrustrated $J$ 's, does not induce an RS phase [54], and a critical strength of frustrated interactions is presumably required to induce it, like in the other frustrated systems [13-16,18-20]. The $Q$ interactions of the $J-Q$ model explicitly favor local correlated singlets and apparently mimic the effects of geometrically frustrated interactions in their ability to generate the RS state.

\section{GROUND-STATE PROPERTIES}

Here we present QMC results for the $J-Q$ model defined in Eq. (1) in the presence of disorder in the form of random $J$ or random $Q$. In most cases, we use a bimodal distribution of couplings $J_{i j} \in\{0, \Lambda\}$ or $Q_{i j k l m n} \in\{0, \Lambda\}$, with equal probability for the two values, but in some cases, we also consider uniform distributions with the couplings bounded by the above values. To contrast random couplings and site dilution, we also consider the $J-Q$ model where a given fraction of the sites, randomly selected, are missing. All operators in Eq. (1) touching one or several missing sites are then removed from the Hamiltonian.

To benchmark our calculations for the $J-Q$ model against a case where it is known that site dilution induces AFM order in a quantum paramagnetic host, we also consider the diluted statically dimerized Heisenberg model illustrated in Fig. 2. In all cases, we average the QMC results over a large number of independent realizations of the disorder (hundreds to thousands) on square lattices with $N=L \times L$ sites and periodic boundary conditions.

Below, in Sec. IVA, we first briefly describe the QMC algorithm used in the ground-state calculations and also introduce the main observables we use to characterize the systems. In the following subsections, we present results for all the models: the diluted $J_{1}-J_{2}$ model in Sec. IV B, the random- $J$ and random- $Q$ systems in Secs. IV C and IV D, respectively, and finally the diluted $J-Q$ model in Sec. IV F

\section{A. Ground-state projector method}

The QMC method we use here projects out the ground state from a trial wave function $|\Psi(0)\rangle$ written in the valence-bond basis consisting of all possible tilings of the square lattice into bipartite singlet bonds. Acting with $(-H)^{m}$ on this state, we obtain an un-normalized state $|\Psi(m)\rangle$; thus, the expectation values of operators $A$ are evaluated in the form

$$
\langle A\rangle=\frac{\langle\Psi(m)|A| \Psi(m)\rangle}{\langle\Psi(m) \mid \Psi(m)\rangle}
$$

for sufficiently large $m$. The different propagation paths contributing to $|\Psi(m)\rangle$ are sampled by expressing $H$ as a 
sum over the $J$ and $Q$ terms in Eq. (1) and carrying out Monte Carlo updates on the corresponding strings (products) of $m$ such operators acting on $|\Psi(0)\rangle$. In this process, the spin d.o.f. are put back in by also sampling the $\uparrow \downarrow$ and $\downarrow \uparrow$ contributions to each valence bond (where one can show that the signs associated with the singlet always cancel out for systems with bipartite interactions) [58]. This way, the projector QMC method in practice becomes very similar to the finite-temperature SSE method [88], with the main difference being that the periodic imaginary-time boundary conditions in the SSE method are replaced by boundary conditions given by the trial state $|\Psi(0)\rangle$. The exact choice of this state is not critical, though a good variational state can improve the convergence rate in $m$ significantly.

The advantage of the projector approach relative to taking the limit $T \rightarrow 0$ in SSE calculations is that the valence bonds restrict the system to the singlet sector (and other sectors can also be accessed by simple modifications). Thus, low-lying $S>0$ states that require very low temperatures to be filtered out in $T>0$ calculations are excluded from the outset. For further technical details on the method, we refer to the literature $[58,87]$.

In the valence-bond basis, expectation values are expressed using transition graphs [93,94] obtained by superimposing the bond configuration from the left and right projected states in Eq. (3). Spin-rotationally-averaged quantities can be expressed using the loops of the transition graphs; e.g., the spin-spin correlation function between two sites $i$ and $j$ vanish if the two sites are in different loops and is $\pm 3 / 4$ for sites in the same loop (with the plus and minus sign corresponding to sites on the same and different sublattices, respectively). Higher-order correlation functions involve more complicated expressions with the transition-graph loops [95].

\section{Order parameters and correlations}

Here we focus on the order parameters of the AFM and VBS phases. The former is the conventional sublattice (staggered) magnetization

$$
\mathbf{M}=\frac{1}{N} \sum_{i=1}^{N}(-1)^{x_{i}+y_{i}} \mathbf{S}_{i},
$$

where the coordinates $x_{i}, y_{i} \in\{0, L-1\}$. Since the simulations do not break the spin-rotation symmetry, we evaluate the expectation value of the squared order parameter $\left\langle M^{2}\right\rangle$, which has a simple loop expression. The VBS order can form with horizontal or vertical bonds, and these are captured by the bond-order parameters

$$
\begin{aligned}
& D_{x}=\frac{1}{N} \sum_{x, y}(-1)^{x} \mathbf{S}_{x, y} \cdot \mathbf{S}_{x+1, y}, \\
& D_{y}=\frac{1}{N} \sum_{x, y}(-1)^{y} \mathbf{S}_{x, y} \cdot \mathbf{S}_{x, y+1},
\end{aligned}
$$

where, for convenience, we switch to a notation where the double subscripts on $\mathbf{S}_{x, y}$ refer to the integer coordinates $\{0, \ldots, L-1\}$ on the square lattice. In this case as well, we need the squared order parameter $\left\langle D^{2}\right\rangle=\left\langle D_{x}^{2}\right\rangle+\left\langle D_{y}^{2}\right\rangle$, which has a reasonably simple direct transition-graph loop estimator [95].

With the above order parameters, we can also define the corresponding Binder cumulants. In the case of the $O(3)$ symmetric AFM order, the proper definition of the cumulant is

$$
U_{M}=\frac{5}{2}\left(1-\frac{3}{5} \frac{\left\langle M^{4}\right\rangle}{\left\langle M^{2}\right\rangle^{2}}\right)
$$

where the coefficients are chosen such that, with increasing system size, $U_{m} \rightarrow 1$ in the AFM phase and $U_{m} \rightarrow 0$ if there is no AFM order. For $\left\langle M^{4}\right\rangle$ as well, there is a simple direct loop expression [95]. In the case of VBS order, the coefficients of the cumulant should be chosen as appropriate for a two-component order parameter, thus,

$$
U_{D}=2-\frac{\left\langle D^{4}\right\rangle}{\left\langle D^{2}\right\rangle^{2}} .
$$

Here, $\left\langle D^{4}\right\rangle$ involves eight-spin correlation functions that in practice are too difficult to compute efficiently [95]. We therefore invoke an approximation in Eq. (7) that does not impact the scaling properties of the cumulant; we simply evaluate $\left(D_{x}, D_{y}\right)$ using the loop estimator for the twopoint operators (5a) and (5b) and then use these classical numbers to evaluate $D^{2}$ and $D^{4}$. While the expectation values entering in Eq. (7) are then not strictly the correct quantum-mechanical expectation values, they still reflect perfectly the absence or presence of VBS order in the system, and $U_{D}$ maintains the desired properties that we discuss above [96].

In addition to the squared order parameters $\left\langle M^{2}\right\rangle$ and $\left\langle D^{2}\right\rangle$ evaluated on the full lattice as we describe above, we also consider the distance-dependent spin and dimer correlation functions,

$$
\begin{aligned}
C_{s}(\mathbf{r})= & \left\langle\mathbf{S}_{x, y} \cdot \mathbf{S}_{x+r_{x}, y+r_{y}}\right\rangle, \\
C_{d}(\mathbf{r})= & \left\langle\left(\mathbf{S}_{x, y} \cdot \mathbf{S}_{x+1, y}\right)\left(\mathbf{S}_{x+r_{x}, y+r_{y}} \cdot \mathbf{S}_{x+1+r_{x}, y+r_{y}}\right)\right\rangle \\
& -\left\langle\mathbf{S}_{x, y} \cdot \mathbf{S}_{x+1, y}\right\rangle^{2},
\end{aligned}
$$

where we spatially average over the reference coordinates $(x, y)$ for each disorder sample. In the case of the spin correlations, we also consider the probability distribution of values without averaging over $(x, y)$ or disorder realizations. The spin correlations have a staggered sign $(-1)^{r_{x}+r_{y}}$, while the sign of the dimer correlator with the $x$-oriented bond as above is $(-1)^{r_{x}}$ (and we take the proper average 
with the $y$-oriented ones). When presenting the results, we remove these signs. In $C_{d}(r)$, it is sometimes better to use the difference between even and odd distances instead of subtracting the squared mean in Eq. (8b).

\section{Spinon strings}

In addition to the physical observables in the singlet sector that we discuss above, it is also useful to consider the lowest state with total spin $S=1$, in which some aspects of spinons can be probed directly. In the valence-bond basis, an $S=1$ state can be expressed with a "broken bond," e.g., with one bond replaced by two $\uparrow$ spins, one each on sublattice $A$ and $B$ (or with one bond treated as a triplet) $[86,97,98]$. These unpaired spins will propagate under the action of the Hamiltonian, and one can characterize their collective nature as bound or unbound, and, in the former case, quantify the size of the bound state [73,98]. Reflecting the nonorthogonality of the valence-bond states, when forming a transition graph out of bra and ket states, the spinons do not have to occupy the same sites in the two states. Referring to the bra and ket sites occupied by the unpaired spins as $a, a^{\prime}$ and $b, b^{\prime}$ on sublattices $A$ and $B$, respectively, open strings of valence bonds will form in the transition graph between $a$ and $a^{\prime}$ and between $b$ and $b^{\prime}$, as illustrated in Fig. 6. The extended nature of the strings reflect the intrinsic size of the spinons [98].

Here we characterize an $S=1$ state by simply using the number of sites involved in the spinon strings. As we see in Sec. IV D, the mean number of sites in the strings scales very differently in the AFM and RS states, and this provides a way, along with other methods, to locate the phase transition between these two states. In addition, in some cases (in Sec. VI), we also use the difference in ground-state energy between the $S=1$ and $S=0$ sectors to extract the spin gap. The spatial distribution of the spinon strings can also give information on the structure of the lowest $S=1$ wave function; this is be investigated in Sec. VI. For technical details on how to carry out the simulations with broken valence bonds, we refer to Refs. $[86,89,97,98]$.

\section{B. Site-diluted $J_{1}-J_{2}$ static dimer model}

We begin our discussion of QMC results with a brief study of a statically dimerized system, where in the uniform system there is a quantum-phase transition from an AFM to a trivial quantum paramagnet due to singlet formation at the stronger bonds. In the case of the columnar model illustrated in Fig. 2, the critical coupling ratio $j_{2 c} \approx 1.91[58,82,83]$. For $j_{2}>j_{2 c}$, it is well known that effective $S=1 / 2$ moments localize around diluted sites in such a system and that these moments interact with each other by nonfrustrated effective couplings mediated by the gapped host system [81], thus, inducing AFM order also in the previously quantum-disordered phase $[84,85]$. Here we use this system as a means of illustrating how this weak-dilution-induced AFM order is manifested in the quantities that we later study in the more interesting (a)

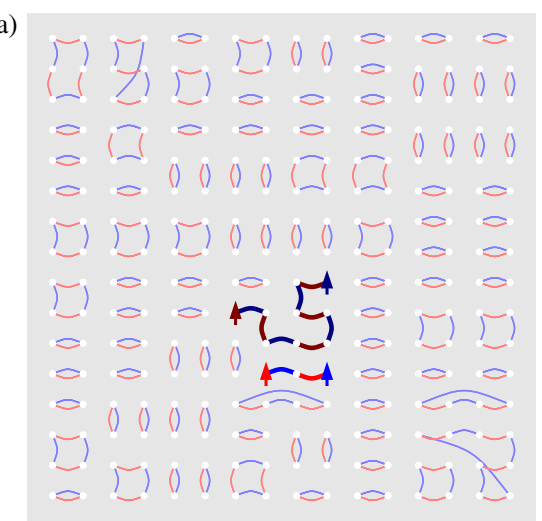

(b)

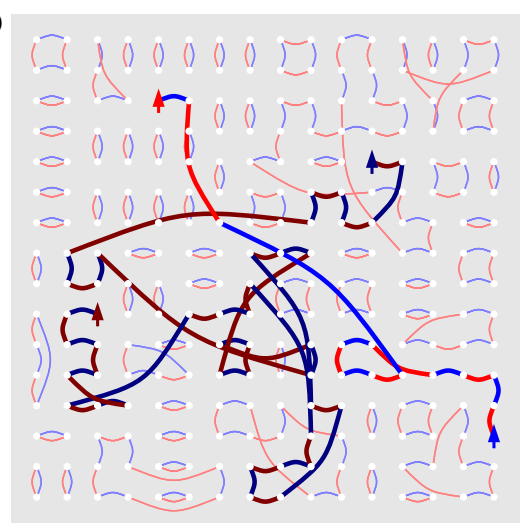

FIG. 6. Transition graphs in the $S=1$ ground state of an $L=16 \mathrm{~J}-Q$ system without disorder in the VBS (a) and AFM (b) phase. For clarity, open boundaries are used here to avoid bonds crossing the boundaries. Red and blue arches correspond to bra and ket valence bonds, with thicker bonds representing the two open strings (depicted in different color shades for clarity) that terminate at unpaired spins (one in the bra and one in the ket state). These end spins are always on sublattice $A$ in one of the strings and $B$ in the other one. In (a), defects in the columnar symmetry-broken VBS pattern originate both from the presence of the spinons and by the intrinsic VBS fluctuations. Formation of a clearly columnar state can be observed only for much larger system sizes [55].

models. For these illustrations, we take the vacancy fraction $p=1 / 32$, with a canonical ensemble such that exactly $N / 32$ sites are removed, with equal numbers on the two sublattices. This density of vacancies is far below the classical percolation threshold $p_{c} \approx 0.407$, beyond which no long-range order can exist.

Figure 7 shows results for both the squared sublattice magnetization and its Binder cumulant. The latter turns out to be a more sensitive quantity for detecting weak order. If there is a critical point separating the AFM phase from a non-AFM phase, the cumulants for two different system sizes, graphed versus the control parameter, should cross each other at a point that drifts toward the critical point with increasing L. However, as shown in Fig. 7(a), the crossing points in this case drift rapidly toward higher $j_{2}$ values, and no convergence with increasing $L$ to a critical coupling can be found. In the inset of Fig. 7(a), the size dependence at 

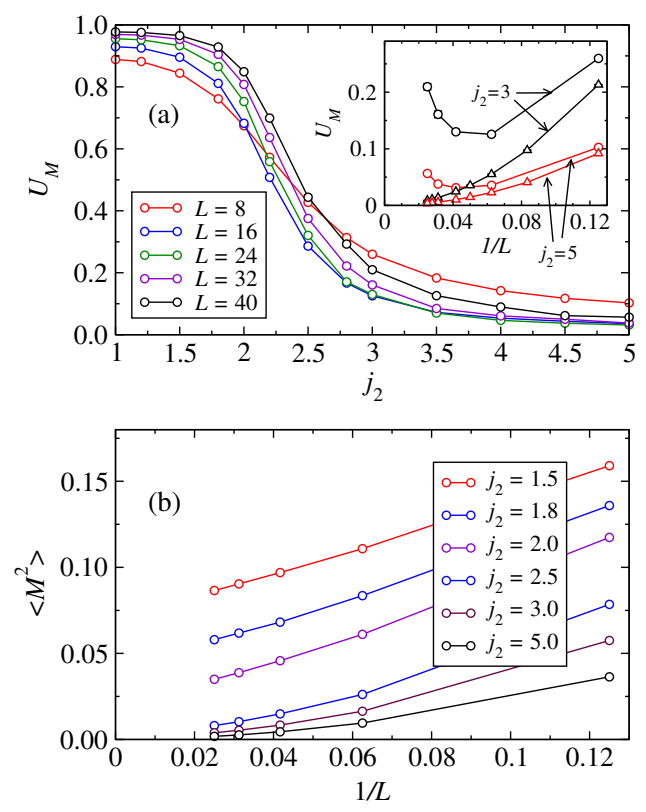

FIG. 7. Results for the diluted $J_{1}-J_{2}$ model at vacancy fraction $p=1 / 32$. (a) AFM Binder cumulant vs the coupling ratio for different system sizes. The inset shows the size dependence for $j_{2}=3$ and 5 for both diluted (circles) and intact (triangles) systems. (b) The size dependence of the squared sublattice magnetization for several values of the coupling ratio. Error bars are smaller than the symbol size in all cases.

two values of the coupling ratio deep inside the quantum paramagnet are shown. Here one can observe nonmonotonic behaviors indicating asymptotic flows toward the value $U_{M}=1$ expected for long-range ordered AFM states. This behavior can be seen even though the order parameter itself shown in Fig. 7(b) is very small. Here all the curves for different $j_{2}$ should extrapolate to $\left\langle M^{2}\right\rangle>0$ when $L \rightarrow \infty$, but for large $j_{2}$, the values are very small and not easy to extract precisely. With the behavior of the Binder cumulants, we can nevertheless confirm that there is long-range order at least up to $j_{2}=5$, and there is no reason to expect any other phase for still larger $j_{2}$.

The reason for the decreasing AFM order with increasing coupling ratio $j_{2}$ deserves some discussion. This behavior can have more than one source and the most important should be the following: (i) The localized moments induce some AFM order in their vicinity, and so each diluted site can contribute effectively more than one unit of staggered magnetization. This effect decreases with increasing $j_{2}$ as the host becomes less susceptible to induced order. (ii) Some of the local moments will form singlets and do not contribute (or contribute very little) to the overall AFM ordering. This effect may also increase with increasing $j_{2}$, as the effective interactions among moments at fixed distance become weaker and the distribution of couplings becomes broader. Therefore, some moment pairs will become more specifically coupled to each other than to other more distant spins in their surroundings. The AFM order cannot be destroyed by these effects, however, as there will always be unpairable moments on sufficiently large length scales, which is supported by previous numerical studies $[84,85]$.

\section{Random- $Q$ model}

We next consider the intact lattice with randomness in the $Q$ interactions, using an extreme case of bimodal coupling distribution where each $Q$ term in Eq. (1) is either absent or present (with equal probability). Here we take the strength of the present six-spin couplings as $2 Q$ so that the parameter $Q$ is the average six-spin coupling. As $Q$ increases, the effective value of the disorder strength, $\Lambda=Q$ in Fig. 5(b), also increases when defined in relation to the constant $J$ coupling. We demonstrate a quantumphase transition between the AFM phase and the phase that we characterize as an RS phase as the coupling ratio $Q / J$ increases. We argue that the phase diagram is of the type schematically illustrated in Fig. 5(b), though we do not consider the full phase boundary versus $\Lambda$. We demonstrate the existence of a quantum-critical point separating the two phases along one path in parameter space and also characterize the ground-state properties of the RS phase in various ways.

\section{VBS domains and apparent lack of AFM order}

First, in Fig. 8, we visualize the VBS domains forming in this kind of system for large $Q / J$, where the pure system is deep inside the VBS phase. Here we observe several instances of meeting points of four domain walls, where spinons are expected to be localized. The clearest example of such a spinon region is indicated by a circle in the lower right corner in Fig. 8. Note that the static dimer pattern, which in Fig. 8 is represented by the nearest-neighbor spin correlations, can be misleading due to the fact that it does not convey completely the quantum fluctuations. A thin line or the absence of a line on a given site implies large fluctuations of the associated spins, as further explained in the caption of Fig. 8, but the nature of those fluctuations is not apparent. Later, in Sec. VI, we also visualize the local spin fluctuations and demonstrate that they are small within the bulk of VBS domains and large at regions corresponding to spinons and domain walls. Despite the possible shortcomings of this type of visualization, it nevertheless makes clear the typical domain size and the manner in which the domains meet. A notable feature is that there are mainly domain walls of the type where the angle $\phi$ (Fig. 3) changes by $\pi / 2$, as would be expected according to the discussion in Sec. III. Some very short segments of $\pi$ domain walls can also be seen, with a line of bonds oriented perpendicularly to those of the adjacent domains located in the gap between those domains. The $\pi$ domain walls in a pure system with a twofold degenerate VBS are gapless with deconfined spinons [92], and in a disordered system with a pinned $\pi$ domain wall one can expect localized 


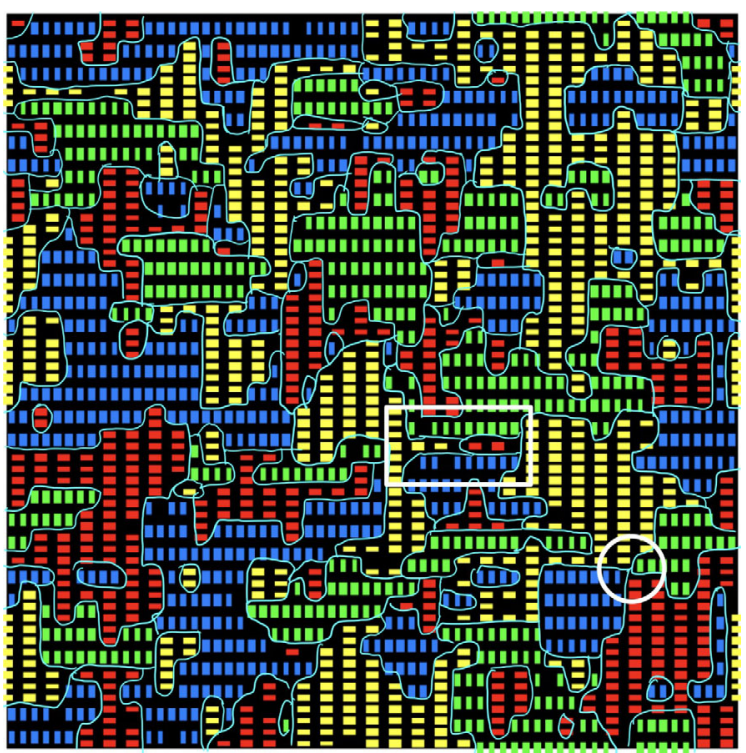

FIG. 8. Visualization of the VBS pattern in the $J-Q$ model generated in simulations with one realization of random bimodal couplings $Q \in\{0,1\}$ and $J=0$ on a periodic $64 \times 64$ lattice. The colored links visualize the corresponding correlations $\left\langle\mathbf{S}_{i} \cdot \mathbf{S}_{j}\right\rangle$ between the spins $i$ and $j$ connected by the link, with the line thickness indicating the magnitude of the correlation. A given link is drawn only if it is the strongest link for both spins $i$ and $j$, and the color coding corresponds to the convention defined in Fig. 3. The domain boundaries are drawn by hand (turquoise curves). The circle indicates an example of a spinon, at which four domain walls meet (as in Fig. 3 but with a different bond arrangement at the nexus of the domains). The rectangle encloses a segment of a $\pi$ domain wall in which two spinons are located.

spinons to form pairwise as well. These spinons can also be regarded as meeting points of four domains, with two of the domains being extremely narrow (chainlike). Examples of local VBS patterns indicative of such spinons can also be seen in Fig. 8 in the form of $\pi$ phase shifts between the VBS patterns of chain segments between two domains. One such domain wall is enclosed by a rectangle in the figure.

The main question now is whether AFM order is induced among the localized spinons that presumably exist in the random VBS environment. We again study the AFM Binder cumulant Eq. (6) as a function of the $Q$ interaction. For convenience, to span the full range of interactions, we graph $U_{M}$ versus $Q /(J+Q)$ in Fig. 9(a). Interestingly, unlike the diluted $J_{1}-J_{2}$ model, Fig. 7 (and also the diluted $J-Q$ model to be discussed later in Sec. IVF), in this case it appears that the cumulants for different system sizes develop a common crossing point as $L$ increases: the standard signal of a quantumphase transition of the AFM state. Furthermore, as shown in Fig. 9(b), for values of $Q / J$ larger than the apparent asymptotic crossing point the cumulants decrease steadily toward zero, and there are no indications of any upturn expected if the state has weak AFM order. One could of course wonder whether a turning point might emerge for
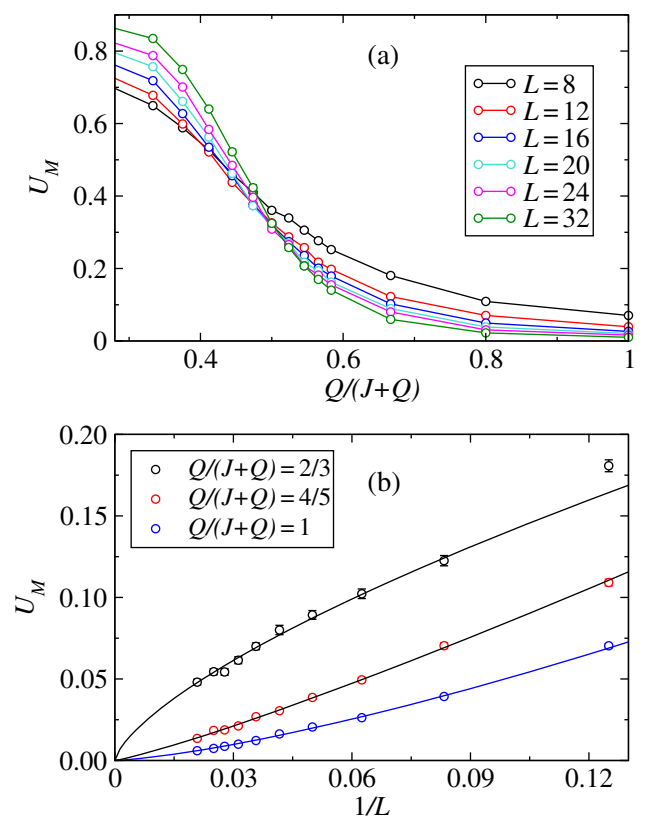

FIG. 9. AFM Binder cumulant of the random- $Q$ model. In (a), the results for several different system sizes are graphed versus the coupling ratio $Q /(J+Q)$, and in (b), the results for three different cases inside the RS phase are graphed vs the inverse system size along with power-law fits.

even larger system sizes, but the very different behaviors of the crossing points between the diluted models, where they drift strongly as the system size increases (as shown in Fig. 7 in the case of the $J_{1}-J_{2}$ model), suggests that the states really are different.

\section{Existence of a phase transition}

The possibility of AFM order for large $Q / J$ in the random- $Q$ model will be excluded if we can convincingly establish the existence of a quantum-critical point where the AFM order parameter and related quantities exhibit critical scaling. To this end, we analyze the drift with $L$ of the cumulant crossing points and also consider an alternative way of locating the critical point.

As we discuss in Sec. IVA, QMC simulations in the valence-bond basis allow also for studies of the lowest triplet state, which is associated with strings representing spinons in the sampled transition graphs (see Fig. 6). In an AFM state, one can expect the spinon strings to cover a finite fraction of the system (and then the spinons are not well-defined particles [98]). We therefore define the string fraction $\lambda$ as the mean fraction of sites covered by one of the spinon strings. In Fig. 10, we demonstrate that, indeed, $\lambda$ approaches a constant when $L$ increases inside the AFM phase, while in the RS phase, $\lambda \propto L^{-1}$. We do not have a rigorous explanation for the latter behavior, but it appears to be a very robust feature of the RS phase. Superficially, it seems to indicate that the spinons are not completely localized but involve of the order of $L$ spins. However, 

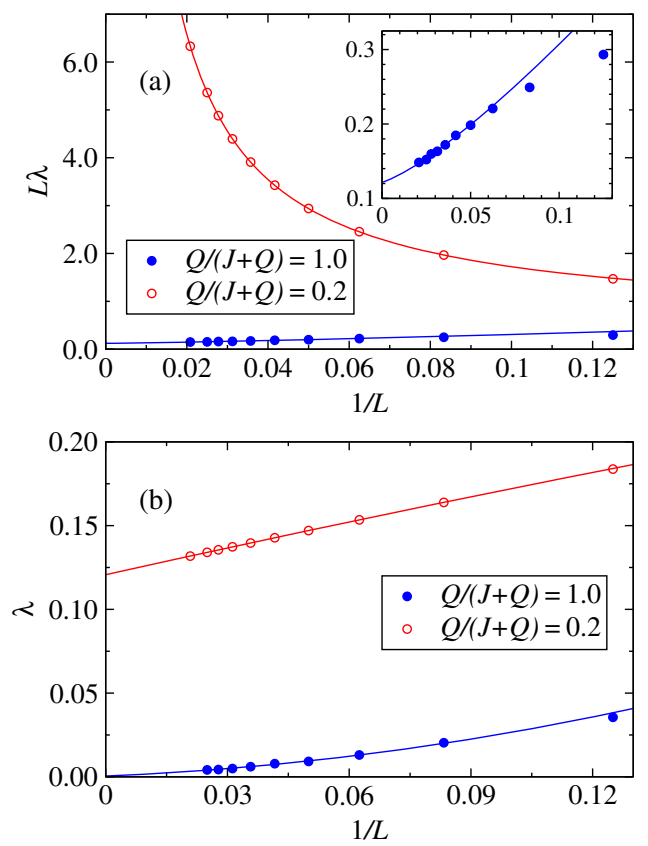

FIG. 10. Finite-size scaling properties of the string fraction $\lambda$ of the random- $Q$ model in the AFM state at $Q /(J+Q)=0.2$ and deep in the RS phase at $Q /(J+Q)=1$. In (a), $\lambda$ is scaled by $L$ to demonstrate $\lambda \propto L^{-1}$ in the RS phase (the inset shows the results on a more detailed scale). The results in (b) illustrate the expected size-independent string fraction in the AFM phase. Error bars are smaller than the symbols.

it should be noted that many spinons can be involved in forming the lowest triplet, and the spinon strings will migrate during the simulations between all of them. The strings then also partially occupy the domain walls (see further discussion of this issue in Sec. VIC), and the mean string fraction is not just probing an individual localized spinon. The precise meaning of the length of the spinon strings in disordered systems should be further investigated; here we merely exploit the apparent utility of $\lambda$ for locating the AFM-RS transition.

Interestingly, as shown in Fig. 11(a), when graphed versus the coupling ratio, $L \lambda$ for different system sizes exhibits crossing points. This would not necessarily be expected when the behavior throughout the RS phase is $\lambda \sim L^{-1}$, but it is still possible due to scaling corrections; indeed, the fact that the crossings occur at smaller relative angles when $L$ increases and all the curves are close to each other for large coupling ratios suggest that corrections to the dominant power law are responsible. While the crossing point is still quite well defined and suggestive of a critical point, the weak size dependence inside the putative RS phase makes it hard to accurately extract the crossing points between curves for system sizes $L$ and $2 L$ when $L$ is large. Nevertheless, we have extracted several crossing points and compare them with the crossing points extracted from Binder cumulant data such as those in Fig. 9. As shown in Fig. 11(b), the size dependence is consistent with flows to a
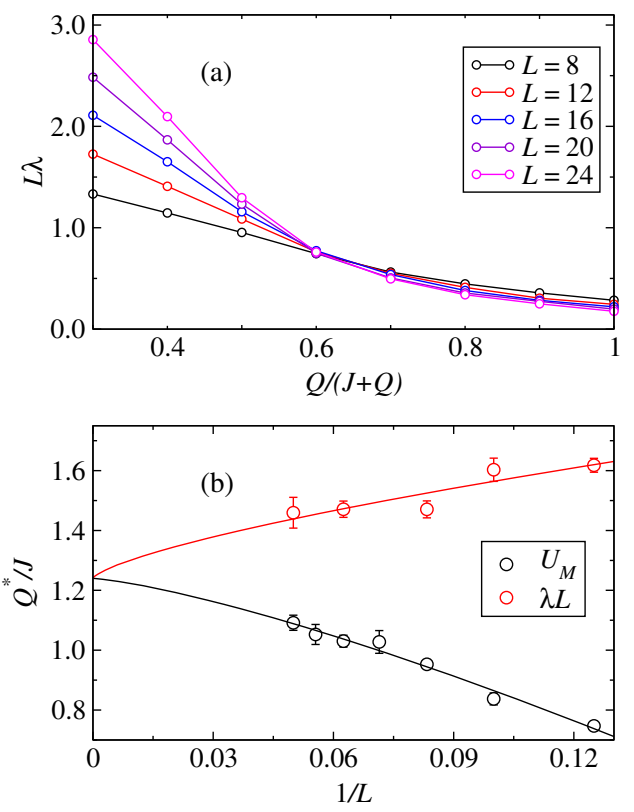

FIG. 11. (a) String fraction multiplied by $L$ vs the coupling ratio $Q /(J+Q)$ of the random- $Q$ model for several system sizes. In (b), crossing points $Q^{*} / J$ extracted from system size pairs $(L, 2 L)$ of data sets such as those in (a) are graphed vs the inverse system size, along with crossing points extracted from the Binder cumulant $U_{M}$ in Fig. 9(a). The curves are fits to a common constant (the critical value $Q_{c} / J=1.24 \pm 0.13$ ) with corrections $\propto L^{-\omega}$, where $\omega \approx 1.4$ and 0.8 for the $U_{M}$ and $\lambda L$ crossings, respectively.

common value (the critical point) when $L \rightarrow \infty$, with power-law corrections in $1 / L$. The two data sets approach the transition from different sides, which is helpful for locating the critical point. We do not have any physical explanation for the different behaviors of the two different data sets, but note that the prefactors of scaling corrections are not universal, and there is no a priori reason to expect that two different finite-size estimates of a critical point should approach it from the same side of the transition.

Since the number of data points for both crossing quantities is rather small, and a common extrapolated $L \rightarrow \infty$ point appears visually very likely, in Fig. 11(b) we carry out a constrained fit with a common infinitesize point. This fit delivers $Q_{c} / J=1.24 \pm 0.13$ (the error bar representing 1 standard deviation). An independent fit to only the $U_{M}$ points gives a fully compatible result, while a fit to only the $\lambda L$ points gives a slightly higher value, $1.4 \pm 0.1$. In the latter case, the number of data points is very small (the number of d.o.f. of the three-parameter fit is only 2), and the error bar is therefore not reliable. Considering the statistically sound joint fit, we take it as strong evidence that both $U_{M}$ and $L \lambda$ are valid indicators of a quantum-critical point separating the AFM phase and a nonmagnetic phase that we argue is an RS phase. 

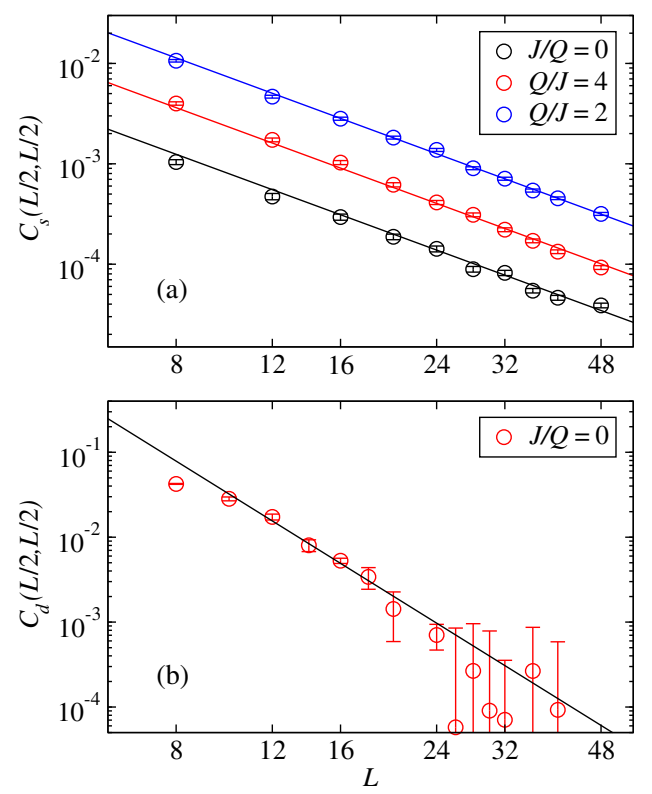

FIG. 12. Absolute values of the mean long-distance spin (a) and dimer (b) correlations at three coupling ratios inside the RS phase of the random- $Q$ model. Results are shown at the largest distance on the periodic $L \times L$ lattices. The three lines in (a) correspond to decay of the form $\propto L^{-2}$, and the line in (b) shows the form $\propto L^{-4}$.

\section{Correlation functions}

Next, we consider the mean spin and dimer correlation functions. Figure 12(a) shows the spin correlations Eq. (8a) at the largest distance on the periodic lattices, $r=L \sqrt{2}$, versus the system size $L$. For three different coupling ratios inside the RS phase, we find the same behavior: a power-law decay corresponding to the distance dependence $C_{s}(r) \propto r^{-\alpha}$ with $\alpha=2$. Instead of carrying out line fits to find $\alpha$, here we just show comparisons with the form with $\alpha=2$, but the individual fits in all cases are also consistent with this value. Interestingly, $C(r) \propto r^{-2}$ is also the form at the RS fixed point in 1D [39], though in that case there are apparently also multiplicative logarithmic corrections [41] that we do not find here in 2D. In the case of the dimer correlations defined in Eq. (8b), Fig. 12(b) shows results at the longest distance where we extract the relevant connected piece of $C_{d}(r)$ as the difference between even and odd distances $r$, which produces less noisy results than the method of subtracting the mean value in Eq. (8b). Here the relative error bars are still rather large for the larger systems, and we show only consistency with the form $C_{d}(r) \propto r^{-4}$, which again is the same form as in 1D (up to the $\log$ corrections found in 1D) [41].

It is also interesting to investigate the probability distribution of the values of the correlation functions in the spatially nonuniform system. Here we again consider the longest distance $r_{i j}=L \sqrt{2}$ on the periodic square lattice and accumulate in histograms all the individual spin correlations $C_{i j}=C\left(r_{i j}\right)$ for spins at sites $i, j$ separated by this distance, with a large number of disorder realizations used to produce reasonably smooth distributions. In this case, it is important to run rather long simulations for each individual disorder realization so that the statistical errors do not influence the distributions significantly for the smaller instances of $C\left(r_{i j}\right)$ (in contrast to the meandisorder-averaged values, where one has only to make sure that the individual simulations are equilibrated, and the final statistical error is dictated by the number of disorder instances). There will always be some problems with large relative errors for the smallest correlations, and therefore, we expect the distributions presented below to be most reliable at the upper end of the distribution.

To investigate the scaling of the distributions, we first attempt a scaling variable similar to one applicable to endto-end spin correlations of the random transverse-field Ising chain, which realizes an IRFP [99],

$$
v=-\ln \left(C_{i j}\right) L^{-a},
$$

and transform the histograms to the distribution $P(v)$. In Ref. [99], the exponent $a=1 / 2$, but here this does not work, and we therefore consider $a$ as a fitting parameter. This indeed works quite well for the larger system sizes if $a \approx 1 / 3$, as shown in Fig. 13(a). We also need the resulting
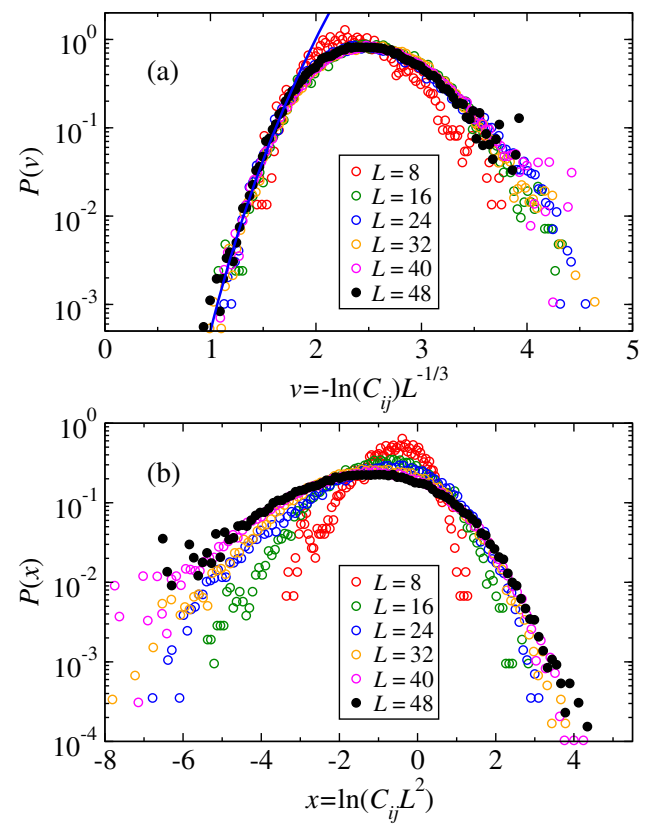

FIG. 13. Two types of histograms showing the distribution of the spin correlations at distance $\mathbf{r}=(L / 2, L / 2)$. In (a), the exponent $a$ in the variable $v$ in Eq. (9) is set to $a=1 / 3$, close to its optimal value for collapse of the data for the larger systems. The blue fitted curve on the left side of the distribution corresponds to the powerlaw behavior $P(v) \propto v^{n}$ with $n=11$. In (b), the scaling variable $x=\ln \left(C_{i j} L^{2}\right)$ is used. 
data-collapsed distribution to be consistent with the mean correlation function,

$$
\left\langle C_{i j}\right\rangle=\int_{0}^{\infty} d v \mathrm{e}^{-v L^{a}} P(v),
$$

for which we previously found $\left\langle C_{i j}\right\rangle \propto L^{-2}$. We can obtain a power law if the behavior of the probability distribution $P(v)$ for the scaled variable $v$ follows a power law close to 0: $P(v) \propto v^{n}$. It is easy to see that the contribution to the mean value from small $v$ then decays as $\left\langle C_{i j}\right\rangle \propto L^{-a(n+1)}$, and with $a=1 / 3$ we therefore need $n=5$. The behavior in Fig. 13(a) is not consistent with this value of $n$, instead giving an exponent $n$ more than twice as large (corresponding to $\left\langle C_{i j}\right\rangle \propto L^{-4}$ ), as shown with a fitted curve in the figure. However, the part of the distribution away from the region where the power law applies still changes the scaling of the mean value to the observed $L^{-2}$ form for the rather small systems we have access to, for which $e^{-v L^{a}}$ in Eq. (10) is not yet very small when $v \approx 2-3$. For large system sizes, the power-law region would always dominate the integral, and with the fitted form we then obtain an $L^{-4}$ decay. Since our data do not extend very close to $v=0$, we cannot exclude that the distribution still changes and evolves into the $v^{5}$ form as $v \rightarrow 0$ and $\left\langle C_{i j}\right\rangle \propto L^{-2}$.

Considering the apparent inconsistencies arising with the scaling variable $v$ above, we explore an alternative form of the distribution. Figure 13(b) shows distributions $P(x)$ with the scaling variable $x$ defined as

$$
x=\ln \left(C_{i j} L^{2}\right) .
$$

In this case, any $P(x)$ trivially gives the desired $L^{-2}$ decay of the mean. Though the data collapse is not as good as in Fig. 13(a), the behavior does seem to improve with increasing $L$, especially at the high end of $x$.

A scaling variable of the form (9) and $P(v) \propto v^{n}$ for small $v$ implies different behaviors of the typical correlations (defined conveniently by the peak of the distribution) and the conventional mean value: exponentially versus power-law decaying. At the IRFP, this behavior is a consequence of the divergent dynamic exponent [99]. As we show in Sec. V, the RS state in the random $J-Q$ model has a finite dynamic exponent, and the scaling with the variable in Eq. (11), which implies the same power-law decay of the mean and typical values, may appear more plausible from this perspective. However, the scaling with the logarithmic variable in Fig. 13(a) works noticeably better, and we cannot exclude that mean and typical values will scale differently even though $z$ is finite. It would clearly be useful to study larger system sizes and further test the two scenarios for the distributions. The inversesquare distance dependence of the mean correlations already appears to be well established by the good scaling for a wide range of system sizes and three different $Q / J$ values in Fig. 12.

\section{Random- $J$ model}

In the random- $J$ model, all $Q$ couplings are included, and the $J$ couplings are drawn from a distribution. We consider bimodal as well as continuous distributions and find qualitatively the same kind of behaviors as above in the random- $Q$ model. We therefore provide only a few illustrative results showing these similarities.

Figure 14 shows the results for the order parameters and Binder cumulants at $Q / J=2$ for the extreme bimodal case where half of the $J$ couplings are set to 0 and the rest to 1 (which we take here as the value of $J$ in the ratio $Q / J$ ). For reference, we compare the size dependence of these quantities with the corresponding pure system (all $J=1$ ). The results indicate that both order parameters vanish when $L \rightarrow \infty$, with the VBS Binder cumulant showing a nonmonotonic behavior with a drop toward zero starting when $L$ is of the order of the typical VBS domain size. For $Q / J=2$, we conclude that the system is in the RS phase.

To confirm the existence of a critical point separating the AFM and RS phases, Fig. 15(a) shows scans for several system sizes of the Binder cumulants versus $Q / J$ for the same bimodal $J$ distribution as in Fig. 14. For $U_{M}$, we again see crossing points apparently converging toward a critical point, similar to the behavior in the random- $Q$ case in
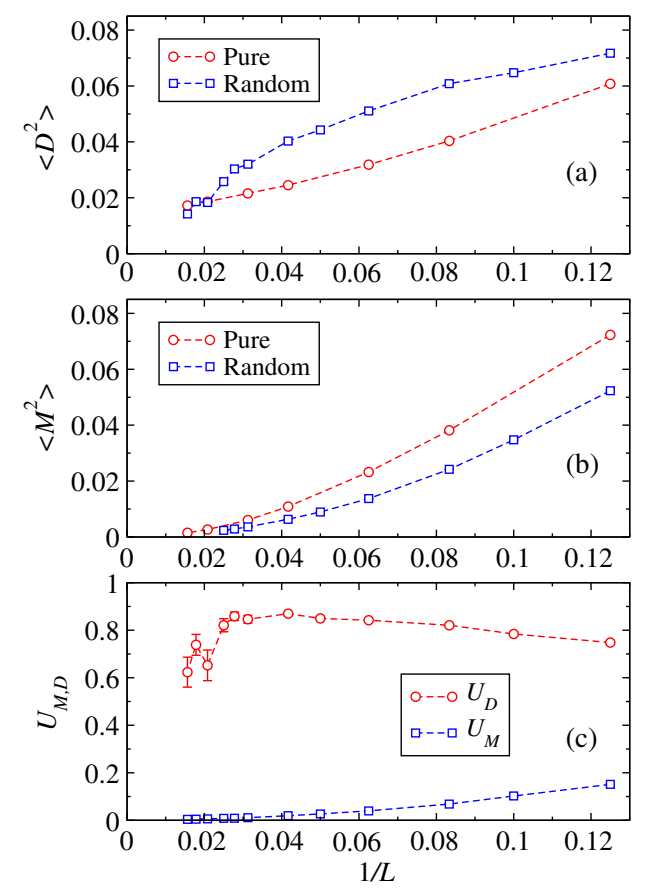

FIG. 14. VBS (a) and AFM (b) order parameters and the corresponding Binder cumulants (c) versus the inverse system size for the model with bimodal $J$ couplings (50\% each of $J=0$ and $J=1$ ) at $Q=2$. Results for the pure model with $J=1$ are shown for comparison in (a) and (b). 

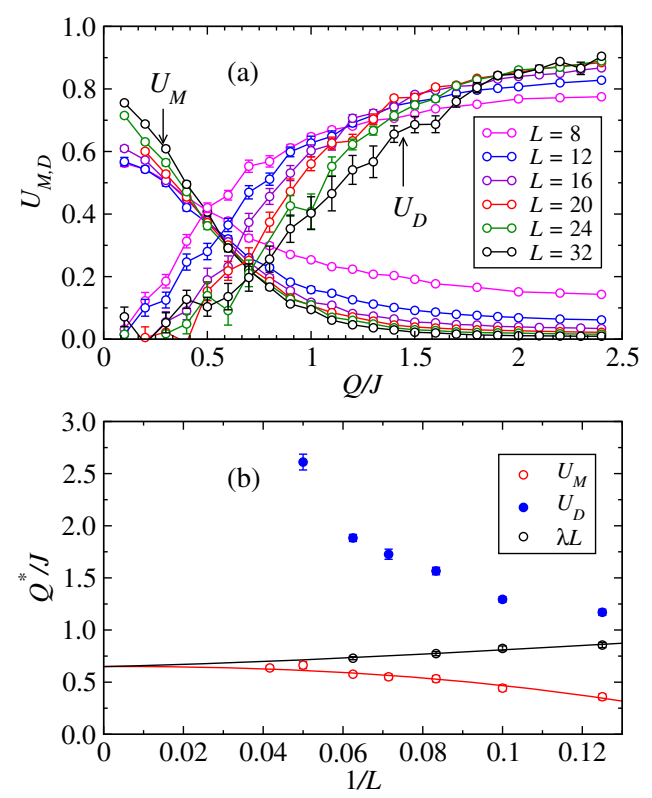

FIG. 15. (a) Binder cumulants vs $Q / J$ for several system sizes of the bimodal random- $J$ model. (b) Crossing points between cumulants for system sizes $(L, 2 L)$ versus $1 / L$. Crossing points of the size-normalized spinon string fraction $L \lambda$ (similar to those shown in Fig. 11) are also shown. Fits (the curves shown) to the latter data set and that for the $U_{M}$ crossing points are carried out using power-law corrections $\propto L^{-\omega}$ (with $\omega \approx 1.5$ and 2.3 for the $U_{M}$ and $L \lambda$ set, respectively), with the constraint of the same value of the crossing point, $Q_{c} / J=0.65 \pm 0.02$, when $L \rightarrow \infty$.

Fig. 9. The $(L, 2 L)$ crossing points are graphed versus the inverse system sizes in Fig. 15(b), along with the crossing points of the scaled string fraction $L \lambda$. These two finite-size estimates of the critical point again approach $Q_{c}$ from different directions. Requiring the fits with corrections $\propto L^{-\omega}$ to have the same value of $Q_{c} / J$ but allowing for different values of $\omega$, we obtain $Q_{c} / J=0.65 \pm 0.02$ and the exponents $\omega \approx 1.5$ (for the cumulant crossings) and 2.3 (for the string quantity). Given the rather small number of points and not very large system sizes, the exponents should be regarded as "effective exponents" that are still influenced by neglected higher-order corrections. Since we have only four $\lambda L$ points in this case, the individual fit to this quantity is not reliable, but an individual fit to the $U_{M}$ data gives results perfectly consistent with the joint fit. Figure 15(a) also shows the behavior of the VBS cumulants. It is clear that the crossing points here do not converge but flow to larger $Q / J$ as the system size increases, as would be expected when arbitrary weak disorder destroys the VBS phase. The corresponding $(L, 2 L)$ crossing points are graphed versus $1 / L$ in Fig. 15(b).

Overall, with the results presented above and in other cases, we find very similar behaviors for the random- $Q$ and random- $J$ models, indicating that the RS phase induced by these types of disorders is the same one. One notable aspect of the specific random- $J$ model for which we present results here is that the RS phase can arise not only out of the VBS phase of the pure model but also from the AFM state. The critical coupling extracted in Fig. 15 is at $Q / J \approx 0.65$, where the pure model with all $J=1$ Heisenberg couplings is still well inside the AFM phase (the AFM-VBS transition of the pure system taking place at $Q / J \approx 1.50$ ). With the way we define the bimodal coupling strengths with $J=0$ and $J=1$ at random locations, we can reach the RS from the AFM phase simply by removing some fraction of the $J$ interactions when $Q$ is between 0.65 and 1.50. This random removal of $J$ couplings enhances the ability of the $Q$ terms to cause VBS formation, which in the random system take only the form of a domain-forming VBS. Thus, it seems very plausible that the same RS state will also be generated if the host system includes some frustrated interactions that weaken the AFM order and favor local formation of VBS domains in a disordered system, instead of the $Q$ terms considered for that purpose here. Such frustrated disordered systems can include the Heisenberg model on the triangular lattice, which is equivalent to the square lattice with half of the diagonal couplings activated. It then appears likely that the RS state we identify here on the square lattice is actually the same state as that discussed previously for frustrated systems. However, further characterization of the frustrated systems is needed to confirm this. We discuss possible scenarios for RG fixed points and flows further in Sec. VII.

\section{E. Universality of the AFM-RS transition}

Given our results presented above, it appears most likely that the AFM-RS transition is universal and that the RS phase itself has universal properties, such as the $1 / r^{2}$ power-law decay of the mean spin correlations (but we show in Sec. V that the dynamic exponent is not universal inside the RS phase but varies continuously - though it also is universal at the AFM-RS transition). An often used characteristic of a critical point is the value of the Binder cumulant. This quantity is universal, in the sense that it is independent on microscopic details, but, unlike many other universal quantities, such as critical exponents, it depends on boundary conditions and aspect ratios of the system [100-102]. In the projector QMC method, we effectively take the limit of the time-space aspect ratio $\beta / L \rightarrow \infty$, and the system geometry is also the same for both the random$Q$ and random- $J$ models. Thus, we have identical boundary conditions and aspect ratios and expect the same value of the Binder cumulant at the AFM-RS transition point.

In Fig. 16, we show results for three disorder types for which we have sufficient data to carry out meaningful studies of the scaling of the AFM cumulant at the $(L, 2 L)$ crossing points; in addition to the bimodal $Q$ and $J$ cases, we also show the results for a continuous distributions of $J$, with values drawn uniformly from the range [0,2]. Remarkably, the cumulants for all cases appear not only to flow to the same point in the limit of infinite size, but even 


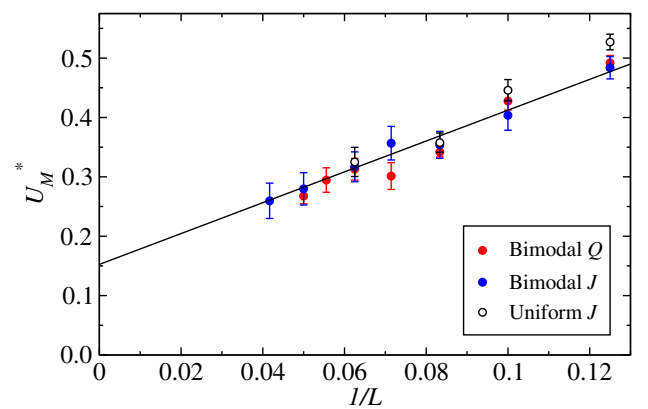

FIG. 16. Binder cumulants vs the inverse system size at the $(L, 2 L)$ crossing points for systems with bimodal $Q$ and $J$ distributions as well as a uniform distribution of $J$ from the range $[0,2]$. The line is a collective fit to the data for the two bimodal cases.

the leading correction in $1 / L$, including the prefactor, seems to be the same. This correction appears to be almost linear, and we analyze the data under this assumption, though it is possible that the form is $L^{-\omega}$ with $\omega$ just close to 1 . For the two bimodal distributions, all the data fall on the line as closely as would be statistically expected (with excellent goodness of fit), while for the continuous distribution, we see that the data for the smaller sizes deviate more significantly, indicating that the higher-order corrections do depend on the kind of the disorder distribution. These results clearly lend further support to the existence of a universal AFM-RS critical point, and, therefore, to the existence of the RS phase.

The slope of the Binder cumulant evaluated at the infinite- $L$ critical point or at crossing points can be used to extract the critical correlation-length exponent $\nu$,

$$
\left.\frac{d U}{d g}\right|_{g=g_{c}}=a L^{1 / \nu}+b L^{1 / \nu-\omega}+\cdots,
$$

where $g$ is the control parameter used, here $g=Q / J$, and $\omega$ is the exponent of the leading scaling correction (and $a, b$ are nonuniversal constants). In practice, it is again convenient to use pairs of system sizes, e.g., $L_{1}=L$ and $L_{2}=2 L$, and replace $g_{c}$ by the crossing point $g^{*}(L)$ of the two cumulants. Then, one can show that (see, e.g., Ref. [73])

$$
\frac{1}{\nu^{*}(L)}=\frac{1}{\ln (2)} \ln \left(\frac{U^{\prime}(2 L)}{U^{\prime}(L)}\right)=\frac{1}{\nu}+c L^{-\omega}+\cdots,
$$

where $U^{\prime}$ denotes the derivative at the crossing point, and $c$ is a nonuniversal constant. Here we obtain the derivatives from the polynomial fits used to interpolate the crossing points from data sets such as those in Fig. 9.

In Fig. 17, we graph the results for $1 / \nu^{*}$ for the same disorder types as in Fig. 16. In the case of the bimodal $J$ and $Q$ distributions, the size dependence is weak, and the results indicate that $\nu \approx 2$. For the weaker, continuous $J$

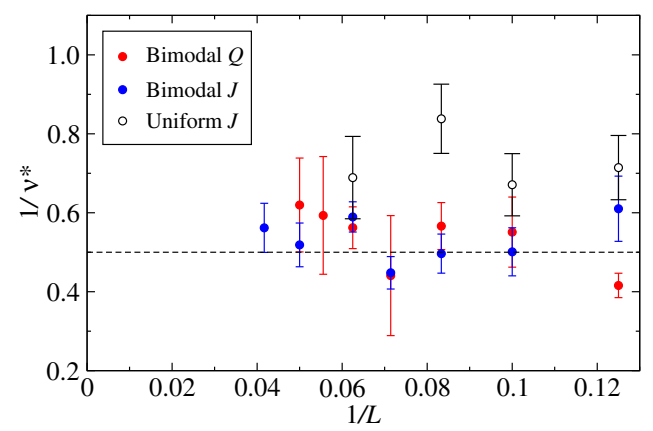

FIG. 17. Inverse system size dependence of the correlation length exponent defined according to Eq. (13). The disorder distributions are the same as those in Fig. 16. The conjectured exponent $\nu=2$ is indicated by the dashed line.

distribution, the values of $1 / \nu^{*}$ are overall larger. However, it is possible that only the bimodal distributions represent strong enough disorder for carrying out reliable extrapolations to infinite size based on the current system sizes, i.e., the corrections to the asymptotic exponent may be larger for the uniform $J$ distribution. An intriguing possibility is that all systems have $\nu=2$, which is also the universal value of this exponent at the $2 \mathrm{D}$ superfluid to Bose-glass transition [33], though the symmetries there are different, and there is no a priori reason to expect the exponents to be the same. Further work is required to test this scenario.

\section{F. Site-diluted $J$ - $Q$ model}

In the site-diluted $J-Q$ model, $J$ or $Q$ terms in Eq. (1) acting on one or more vacancies are excluded from the Hamiltonian. We consider small vacancy concentrations $p$ and always remove an equal number of sites on the two sublattices. In the gapped VBS host, when $Q>Q_{c}$, with $Q_{c} / J \approx 1.50$ [55], we expect the vacancies to act as nucleation centers for VBS vortices [90]. Here, no spinon will appear in the VBS vortex core as there is an empty site. However, with the random distribution of the vacancies, there will be local sublattice imbalance, i.e., an unequal number of vortex cores on the two sublattices; within a group of $n$ vacancies, there will be an imbalance of order $\sqrt{n}$ that makes impossible the short-distance pairing of all vacancy vortices and antivortices. Therefore, additional vortices will form away from the vacancies, and these topological defects will source unpaired spins (spinons). There are reasons to believe that these spinons cannot be paired up into singlets in the way this happens in the RS state because of the local imbalance between $A$ and $B$ spinons. The mechanism of primarily short-distance pairing responsible for the RS state is, thus, missing, and AFM order should form as in the $J_{1}-J_{2}$ model studied in Sec. IV B.

The results for $p=1 / 32$ at two different values of the coupling ratio are shown in Fig. 18. Here in Fig. 18(a), we can again see, as we did in the case of the $J_{1}-J_{2}$ model in Fig. 7, how the AFM Binder cumulant first decreases with 


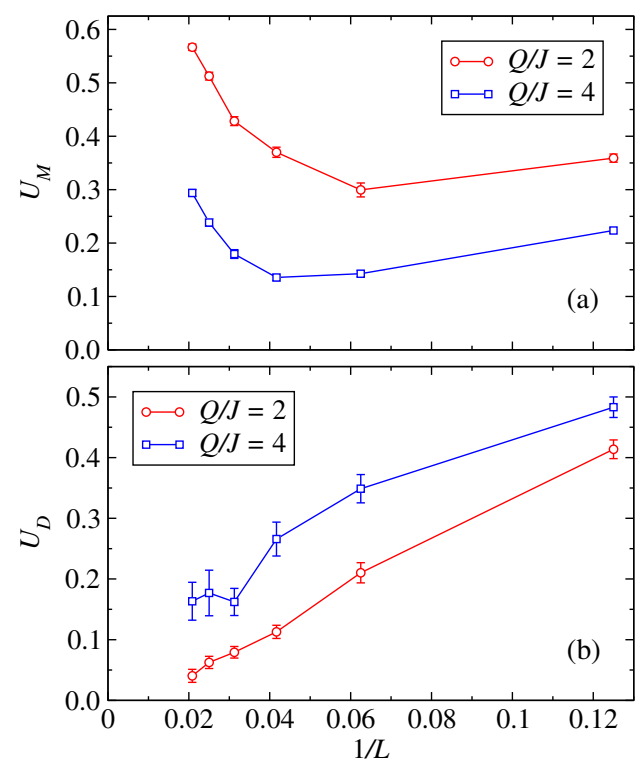

FIG. 18. Binder cumulants of the AFM (a) and VBS (b) order parameters of the site-diluted $J-Q$ model at $Q / J=2$ and 4 , graphed vs the inverse system size. The dilution fraction is $p=1 / 32$, with exactly half of the vacancies in each sublattice.

increasing system size but then starts to grow when the number of moments becomes sufficient for AFM order to form. This crossover occurs for larger sizes for the larger $Q / J$ value, which is again similar to the behavior found for increasing coupling ratio $J_{2} / J_{1}$ in the $J_{1}-J_{2}$ model. Figure 18(b) shows that the cumulant of the dimer order parameter approaches zero with increasing $L$, as expected for a VBS breaking up into domains. These results lend support to a phase diagram of the type in Fig. 5(a), with no phase transition for $\Lambda>0$, just a crossover between strong and weak AFM order.

\section{FINITE-TEMPERATURE PROPERTIES AND THE DYNAMIC EXPONENT}

Finite-temperature properties are useful for extracting the dynamic exponent $z$ and may be the most direct route to connect to experiments. Here, we consider the uniform magnetic susceptibility,

$$
\chi_{u}=\frac{1}{T N}\left\langle m_{z}^{2}\right\rangle, \quad m_{z}=\sum_{i=1}^{N} S_{i}^{z},
$$

and the local susceptibility at location $\mathbf{x}$ defined by the Kubo integral

$$
\chi_{\mathrm{loc}}(\mathbf{x})=\int_{0}^{1 / T} d \tau\left\langle S_{\mathbf{x}}^{z}(\tau) S_{\mathbf{x}}^{z}(0)\right\rangle,
$$

where $S_{\mathbf{x}}^{z}(\tau)$ is the standard imaginary-time-dependent spin accessible in QMC simulations. Here we use the SSE method and refer to the literature, e.g., Ref. [58], for further technical information. In this section, we average the local susceptibility over all the sites $\mathbf{x}$ of the system (as well as over disorder realizations) and call this averaged quantity $\chi_{\text {loc }}$. In Sec. VI, we show an example of the spatial dependence of $\chi_{\text {loc }}(\mathbf{x})$ for a fixed disorder realization.

\section{A. Power-law behaviors}

At a conventional quantum-critical point, or in an extended quantum-critical phase, since the magnetization is a conserved quantity, the susceptibility Eq. (14) should scale with the temperature as $[33,103]$

$$
\chi_{u} \propto T^{D / z-1}
$$

where $D=2$ in our case. In contrast, the local susceptibility Eq. (15) is sensitive to the fluctuations of the nonconserved critical order parameter. Generalizing the result by Fisher et al. [33] for a critical point of a disordered boson system (the Bose glass to superfluid point) to the critical RS phase, the mean spin-spin correlation function in imaginary time at zero spatial separation should have the form

$$
\left\langle S_{\mathbf{X}}^{z}(\tau) S_{\mathbf{x}}^{z}(0)\right\rangle \propto \tau^{-(D+z-2+\eta) / z}=\tau^{-2 / z},
$$

where in the equality we use our finding that the equal-time correlation function $C(r) \propto r^{-(D+z-2+\eta)}$ [33] always decays with distance as $1 / r^{2}$ so that $\eta=2-z$. The local susceptibility (15) is then predicted to take the following forms

$$
\chi_{\mathrm{loc}}= \begin{cases}a+b \ln (1 / T), & \text { for } z=2, \\ c T^{2 / z-1}, & \text { for } z>2\end{cases}
$$

with nonuniversal constants $a, b, c$. Here and in Eq. (16), it is interesting to note that the uniform and local susceptibilities should take the same divergent form if $z>2$, while for $z=2$, the logarithmic divergence in $\chi_{\text {loc }}$ is not present in $\chi_{u}$, which instead should be temperature independent (up to possible additive corrections).

For the above forms of $\chi_{u}$ and $\chi_{\text {loc }}$ to be valid, we not only have to reach sufficiently low in $T$, but also the system size has to reach the range where there is no longer any size dependence left. This requirement limits the temperatures we can reach, as demonstrated in Fig. 19 for the case of the uniform susceptibility of the random- $Q$ model close to the critical point and inside the RS phase. We can still clearly observe critical behaviors emerging for a range of low temperatures for the largest system sizes. In Fig. 19(a), at $Q / J=1.20$, which should be very close to the AFM-RS transition according to the results in Fig. 11(b), we find very little temperature dependence (except for the lowest temperatures, where there are still clearly some effects of finite size), indicating, by Eq. (16), that $z=D=2$ at the transition. In principle, one should also expect some 

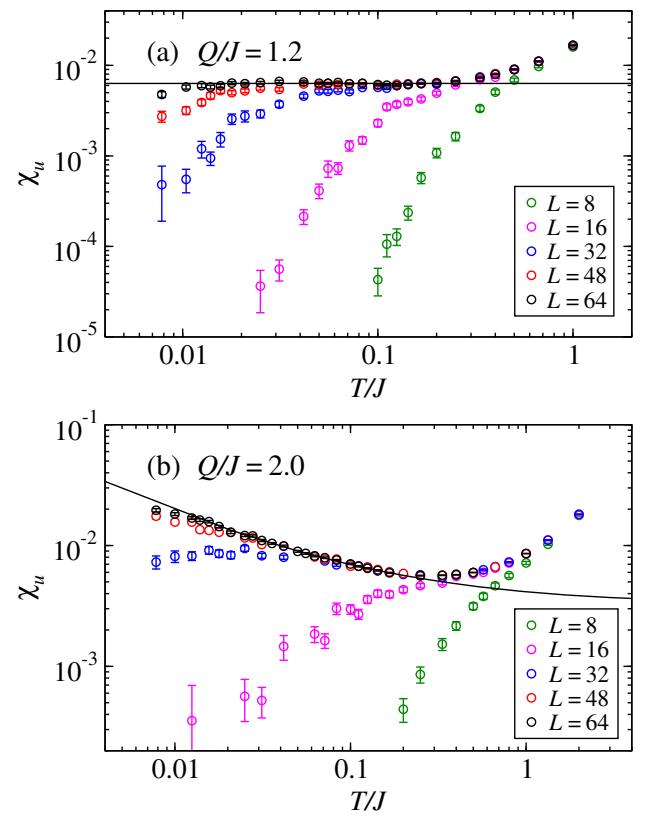

FIG. 19. Temperature dependence of the uniform susceptibility of the random- $Q$ model for several different system sizes. (a) shows results at $Q / J=1.2$, which is within the error bars of the estimated AFM-RS critical point (Fig. 11), while the system in (b) is inside the RS phase at $Q / J=2$. The horizontal line in (a) corresponds to the scaling expected if the dynamic exponent $z=2$. The curve in (b) shows a fit of the $L=64$ data to the form $\chi_{u}=c+b T^{-a}$ with the exponent $a=0.60 \pm 0.08$ corresponding to $z=2 /(1-a) \approx 5$.

corrections to the constant behavior, but apparently those are very small in this case.

If the dynamic exponent at the AFM-RS transition indeed takes the value $z=2$, then according to Eq. (18) the local susceptibility should exhibit a logarithmic divergence. As shown in Fig. 20, this indeed appears to be the case. Here we fit the low- $T$ behavior to the first line in Eq. (18), which already contains a constant (unlike the uniform susceptibility in Fig. 19, where we include a constant as a correction to the leading form).

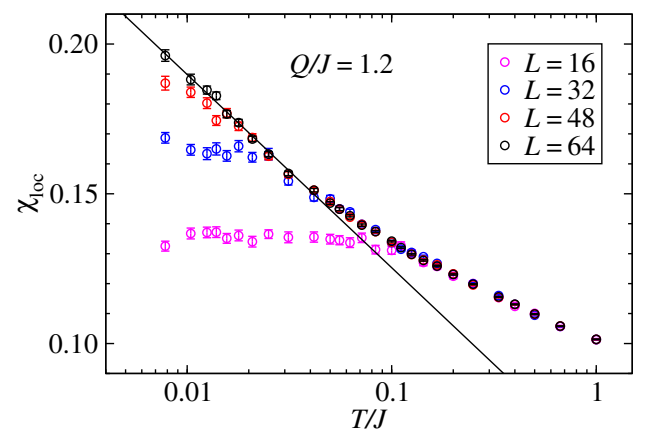

FIG. 20. Temperature dependence of the local susceptibility of the random- $Q$ model at its AFM-RS transition for several different system sizes. The line is a fit of the $L=64$ low- $T$ points to the logarithmically divergent form in Eq. (18).
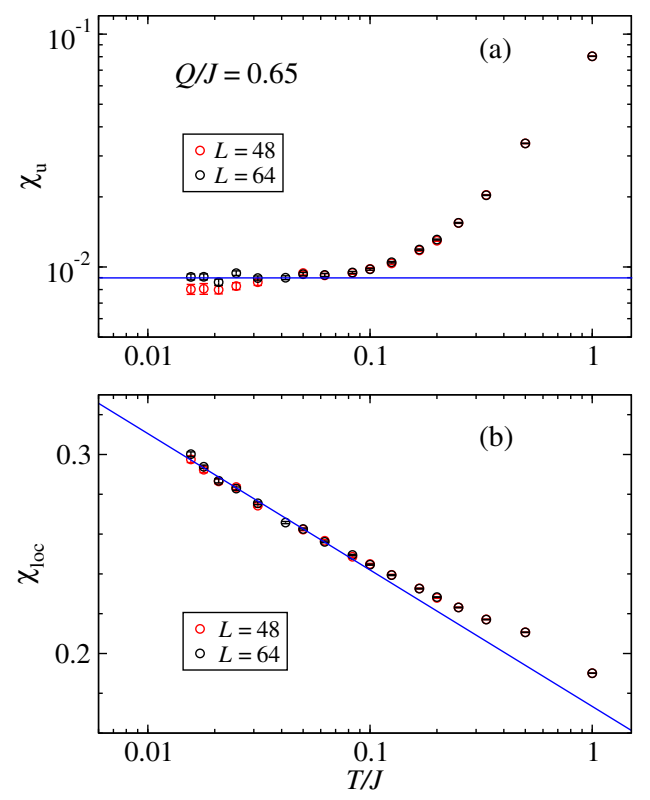

FIG. 21. Temperature dependence of the uniform (a) and local (b) susceptibility of the bimodal random- $J$ model at its estimated critical point $Q_{c} / J \approx 0.65$ [see Fig. 15(b)]. The horizontal line in (a) indicates the size-independent behavior expected from Eq. (16) if $z=2$, while the line in (b) is a fit to logarithmic form in Eq. (18).

To test the universality of the scaling of the susceptibilities at the transition, in Fig. 21 we show the results for the bimodal random- $J$ model at its critical point extracted in Fig. 15(b). We compare results for two different system sizes to demonstrate that the thermodynamic limit should be reproduced for the larger size $(L=64)$. We again see a significant low- $T$ regime where $\chi_{u}$ appears to be temperature independent, while the local susceptibility diverges logarithmically, supporting an AFM-RS transition with $z=2$ independent of model details.

Well inside the RS phase, at $Q / J=2$ in the random- $Q$ model, as shown in Fig. 19(b) we find a clearly divergent low- $T$ behavior of $\chi_{u}$. Since the overall magnitude of the susceptibility originating from the localized spinons is still not very large at these temperatures, when fitting to the expected power-law form, we also include a constant, as a natural leading correction to the asymptotic divergent form. This works well, and the value of the exponent given by the fit corresponds to $z \approx 5$. Thus, we find that $z$ increases as the RS phase is entered.

Figure 22 shows results even further inside the RS phase, along with fits such as those discussed above. Here we also show results for the local susceptibility, which at first sight appears to diverge slower, though the ultimate power laws should be the same if $z>2$, according to Eqs. (16) and (18). However, with independent constant corrections added, both $\chi_{u}$ and $\chi_{\text {loc }}$ can be fitted with the same power laws (using joint fits). These fits give the exponent 

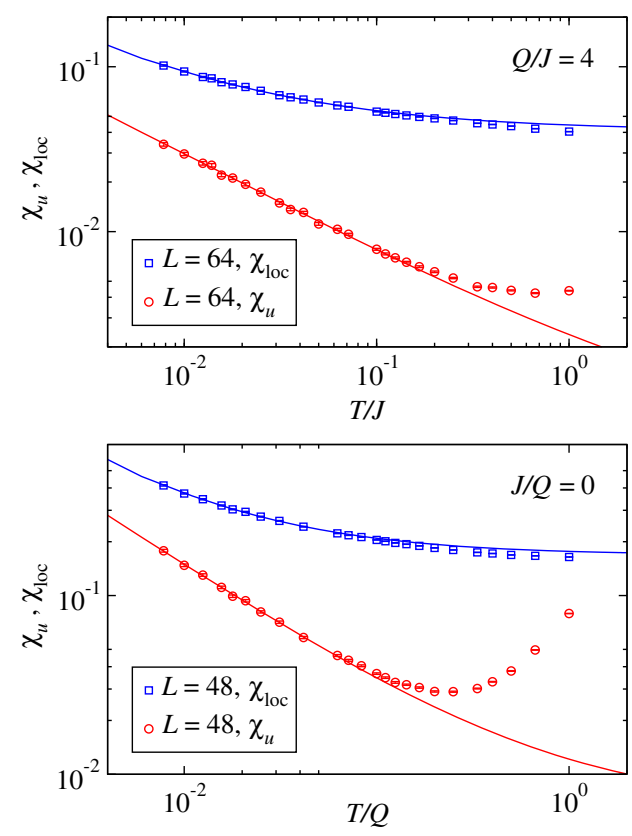

FIG. 22. Temperature dependence of the uniform and local susceptibilities of the random- $Q$ model deep inside the RS phase, at $Q / J=4$ and $J / Q=0$. The curves show fits of the low- $T$ data to the predicted forms, Eq. (16) for $\chi_{u}$, and the second line of Eq. (18) for $\chi_{\text {loc }}$, enforcing common exponents for the two quantities. The fits give $1-2 / z=0.60 \pm 0.10(Q / J=4)$ and $0.72 \pm 0.06(J / Q=0)$.

$1-2 / z=0.60 \pm 0.10$ and $0.72 \pm 0.06$ for $Q / J=4$, and $J / Q=0$, respectively; i.e., the dynamic exponents are $z \approx 5$ at $Q / J=4$ and $z \approx 7$ when $Q / J \rightarrow \infty$.

In the case $J=0$, it should be noted that the bimodal disorder distribution that we use here, where half the $Q$ couplings are set to zero, can lead to isolated spins that contribute $\propto 1 / T$ to the susceptibility. However, we avoid this issue by "patching" such rare isolated spins by adding a randomly chosen $Q$ interaction for each of those sites to connect them to the rest of the system. The differences between the patched and original systems are barely noticeable in the disorder-averaged quantities.

The larger role of the correction (the constant terms used in the fits in Fig. 22) in $\chi_{\text {loc }}$ than in $\chi_{u}$ can likely be traced to the fact that the local susceptibility only contains a small fraction of the dominant staggered response at $q=(\pi, \pi)$ in momentum space, and, therefore, one may expect large corrections from all the other momenta, at which the response is weaker. An alternative way to detect the dominant dynamic response, but that we have not yet pursued, would be to compute the susceptibility at $q=(\pi, \pi)$.

\section{B. Griffiths-McCoy singularities}

To properly classify the proposed RS state, we need to consider the fact that disordered systems generically have regions in parameter space called Griffiths, or GriffithsMcCoy, phases. These phases or regions are characterized by spatial "comingling" of two phases [104,105]. Fluctuations in the quenched disorder can favor a phase $B$ within a limited part of a system that is overall in a phase $A$. Griffiths phases, which do not always have well-understood RG fixed-point analogues (but sometimes they do [106]), appear close to critical points and are normally associated with weaker singularities than the actual critical points (for reviews, see Refs. $[27,28])$. The singularities arise from exponentially rare regions (e.g., large domains of phase $B$ inside phase $A$ ) and have the most profound effects on dynamical properties.

In quantum systems, Griffiths phases typically have large but finite dynamic exponents, with associated divergent susceptibilities if $z>D$. The large $z$ values (long timescales) motivate the often used term "glass" for these phases, though a Griffiths phase is not normally associated with the multitude of thermodynamic states (by replica symmetry breaking and related phenomena) of classical and quantum-spin glasses (and it was also claimed that the valence-bond glass state undergoes replica symmetry breaking [77], but this may be a consequence of a classical treatment). Examples of Griffiths phases include the Bose glass in the Bose-Hubbard model with random potentials [33] and the Mott glass in particle-hole symmetric boson systems where randomness is introduced in the hopping (and there are indications that this state can also form with random potentials due to emergent particle-hole symmetry [107]). The spin analogue of particle-hole symmetry is also present in 2D random-exchange Heisenberg antiferromagnets, where Mott-glass phases have been identified $[108,109]$.

An important question is whether the RS state we identify in the random $J-Q$ model is also a Griffiths phase. We argue that it is not because equal-time correlations in Griffiths phases should decay exponentially with distance (a fundamental consequence of the rare-region mechanism), while we find strong evidence for power-law decaying correlations.

There is a further strong argument against the RS phase being a Griffiths phase: If, in the language above, we consider the AFM as phase $A$, there is no obvious phase $B$ with which $A$ can comingle to form the RS phase as a Griffiths phase. The RS phase is then actually that phase $B$, and, in principle, Griffiths singularities could appear due to comingling of the AFM and RS phases close to the phase boundary. However, since the AFM and RS phases are both gapless, the Griffiths singularities would be very hard to detect and would very unlikely be responsible for the power laws we identify here. Most likely, they would cause only scaling corrections and no separately identifiable Griffiths phase in addition to the AFM and RS phases.

RS states and Griffiths phases have been contrasted in detail in Heisenberg chains [110]. In a chain with the same disorder distribution on all links, the RS state forms generically. However, if there is furthermore an alternating strength of the mean couplings (static dimerization), in which case the pure system is gapped and has exponentially decaying correlations, a critical disorder strength is required to induce 
the RS state. The weakly disordered system is in a Griffiths phase, where rare RS regions in the otherwise gapped chain imply gapless excitations of the system. However, the spin correlations in this phase remain exponentially decaying. The RS state itself is not a Griffiths phase.

\section{SPINON INTERACTION MECHANISM}

The way the localized spinons interact with each other is a crucial ingredient in the formation of the RS state. In order for singlets to be gradually "frozen out" as the energy scale is reduced (as in the Ma-Dasgupta strong-coupling RG procedure $[38,46])$, and for AFM order not to form on large length scales, in 2D it seems necessary that the spinon-spinon interactions are not completely random. This scenario is evidenced by the fact that 2D SDRG calculations on $S=1 / 2$ systems with various coupling distributions have so far not been able to generate a phase similar to the RS phase identified here [36,37]. In the $J-Q$ model, the observation that spinons are created in pairs (spinons and antispinons) when VBS domains are formed already implies a correlation that favors closer typical distance between a spinon and the nearest antispinon because the domain walls will provide an effective attractive potential due to the domain-wall energy increasing with distance between a spinon and antispinon site connected by a wall. There is, however, potentially also another effect; namely, the effective magnetic interactions between the spinons are likely mediated mainly through the domain walls. The putative role of domain walls as mediators of spin correlations was mentioned in Ref. [18] but was not developed into an actual mechanism suppressing AFM order and causing the singlet formation in the RS state. Here we provide evidence for such a mechanism within our models on the square lattice. We note that the effective interactions should have the same bipartite nature as the microscopic interactions in the pure system, as was discussed generically in Ref. [18] (and earlier in specific cases, e.g., in Ref. [111]).

\section{A. Uniform domain wall}

First, let us consider a uniform domain wall in the pure $J-Q$ model in its VBS state. According to the DQC theory [74], the thickness of a domain wall between VBS domains, across which the angle $\phi$ defined in Fig. 3 changes by $\Delta \phi=\pi / 2$, is not governed by the standard correlation length $\xi$ but by a longer length scale $\xi^{\prime}$ (i.e., this length diverges faster than $\xi$ as the DQC point is approached). This second length affects the scaling of the energy density of the domain wall as the critical point is approached [75,89], which may also have a counterpart at the AFM-RS transition. We only mention this here and do not explore the domain-wall thickness further. Instead, we discuss the spin gap of a domain wall, i.e., the energy difference between the $S=0$ ground state and the lowest $S=1$ state in a system with a domain wall imposed by boundary conditions.

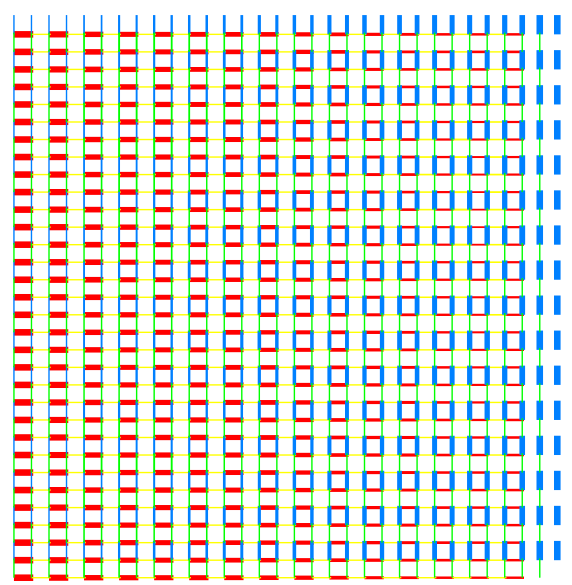

FIG. 23. Domain wall on a $32 \times 32$ lattice for a system with $J=0, Q=1$. The bonds are colored according to the convention in Fig. 3, and the line thickness represents the expectation value $-\left\langle\mathbf{S}_{i} \cdot \mathbf{S}_{j}\right\rangle$. The left and right open boundaries are modified at the Hamiltonian level to lock in VBS patterns differing in angle by $\Delta \phi=\pi / 2$. Note that a $2 \times 2$ plaquette with equal correlation on all edges seen in the middle of the system corresponds to the VBS angle $\phi=\pi / 4$.

Figure 23 shows an example of a domain wall, where the bond thickness on a $32 \times 32$ lattice corresponds to the magnitude of the spin correlation on that bond, and the colors of the bonds are coded as in Fig. 3. The boundary conditions are periodic in the vertical direction, but in the horizontal direction the interactions are modified (see Ref. [89]) so that the edges are locked into VBS realizations differing by the angle $\Delta \phi=\pi / 2$ of an elementary domain wall. Here it should be noted that the length scale over which the angle $\phi$ changes in Fig. 23 is not the intrinsic domain-wall width because the location of the wall also has quantum fluctuations that smear it out when the expectation values are computed. The spin gap of the wall is still a completely well-defined quantity, as long as the $S=1$ excitation (observed, e.g., with the spinon strings illustrated in Fig. 6) is not repelled from the wall. We have confirmed that the excited spin is attracted to the domain wall (which by itself implies, by energy minimization, a smaller gap on the wall than in the bulk VBS away from the wall).

The spin gap is obtained by simply taking the difference between total ground-state energies computed in the two spin sectors. Figure 24 shows the results for the uniform system without domain walls (obtained with fully periodic $L \times L$ lattices) and with domain walls on lattices with two different aspect ratios, as a test of the expected independence on the lattice geometry when $L \rightarrow \infty$. For small systems with a wall, the gap is strongly influenced by the boundary modifications, which here extend three rows into the system on each side, and one should not draw any conclusions on the differences between the system with and without the domain wall until $L$ is much larger and the wall has converged to its intrinsic thickness. For large $L$, it is 


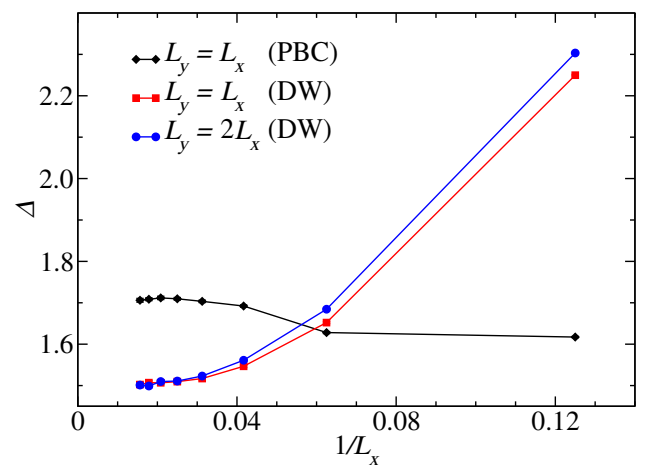

FIG. 24. Singlet-triplet gaps of the nonrandom system at $J=0$, $Q=1$ with and without a domain wall. The bulk VBS gap corresponds to the $L \rightarrow \infty$ limit of the results for the system with periodic boundaries. For systems with a domain wall (illustrated in Fig. 23) induced by modified open boundaries in the horizontal direction, two different aspect ratios $L_{y} / L_{x}$ are considered as a check of a unique intrinsic gap of the domain wall when $L_{x} \rightarrow \infty$.

clear that the gap on the domain wall $\Delta / Q \approx 1.5$ is significantly smaller than in the bulk $\Delta / Q \approx 1.7$, as one might expect just from the fact that the domain wall has weaker order, i.e., more fluctuations, than the bulk VBS. Thus, in a nonrandom system, a domain wall will be a more effective mediator of correlations, and thereby of effective interactions between impurity spins, than in the bulk VBS.

\section{B. Local susceptibility}

The above results for a pure infinitely long domain wall should be taken only as suggestive of enhanced spinon interactions along domain walls in the disordered system. We can obtain further evidence by examining the spatial variations of the local susceptibility, Eq. (15), for individual disorder realizations (see Ref. [97] for similar calculations for a diluted Heisenberg system). A large susceptibility can be taken as a sign of a small local gap through the sum rule (here written only for $T=0$ )

$$
\chi_{\mathrm{loc}}(\mathbf{r})=2 \int_{0}^{\infty} d \omega \omega^{-1} S_{\mathrm{loc}}(\mathbf{r}, \omega),
$$

where $S_{\mathrm{loc}}(\mathbf{r}, \omega)$ is the local dynamic spin structure factor, which satisfies another sum rule,

$$
\int_{0}^{\infty} d \omega S_{\mathrm{loc}}(\mathbf{r}, \omega)=S_{\mathrm{loc}}(\mathbf{r})=\left\langle S_{\mathbf{r}}^{z} S_{\mathbf{r}}^{z}\right\rangle=\frac{1}{4} .
$$

For any finite system, the spectral weight in $S_{\text {loc }}(\mathbf{r}, \omega)$ does not extend all the way down to $\omega=0$, and in a single-mode approximation, where there is only a single $\delta$ function at $\omega=\Delta$, we can extract the local gap as $\Delta(\mathbf{r})=2 S_{\text {loc }}(\mathbf{r}) /$ $\chi_{\mathrm{loc}}(\mathbf{r})=\left[2 \chi_{\mathrm{loc}}(\mathbf{r})\right]^{-1}$. In the realistic case where there is a broader distribution of spectral weight, $\chi_{\mathrm{loc}}(\mathbf{r})$ can still be regarded as a proxy for the typical local low-energy scale, and it should then also be a measure of the local ability

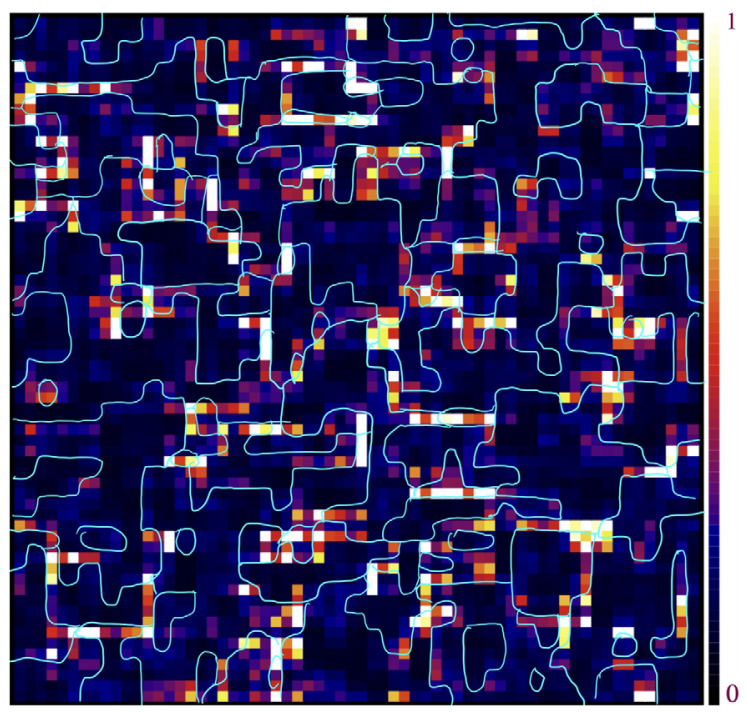

FIG. 25. Visualization of the local susceptibility for the same coupling realization of the random- $Q$ model for which the VBS domains are illustrated in Fig. 8 and with the same hand-drawn domain boundaries (turquoise curves). The values of the susceptibility defined in Eq. (15) are rescaled so that the maximum is 1, and the color coding is shown on the bar on the right side. Note that in some cases, a nexus of four domain walls is dark because it does not correspond to a spinon. This can be seen by comparing with Fig. 8, where it can be seen that the cases in question involve only two different domains (thus, no spinon is present).

of a region of the system to mediate effective spin-spin interactions.

In Fig. 25, we show the spatial dependence of the local susceptibility for the same $Q$ disorder realization as in the illustration of VBS domains in Fig. 8. Several bright spots on the susceptibility map can be observed, and many of them can be matched with meeting points of four VBS domain walls, where spinons should localize. Naturally, the sites on which the spinons reside should have enhanced susceptibility (and note that a single spinon will be spread out over several sites due to quantum fluctuations). There are also bright regions in Fig. 25 along many of the domain walls, while in the bulk of large VBS domains, there are no bright spots. These observations support the notion that the domain walls act as mediators of spinon-spinon interactions, which should play an important role in the formation of the RS state.

\section{Spinon strings}

As seen in Eq. (19), the local static susceptibility represents an inverse-frequency weighted average over a local dynamic response function. By selection rules, the relevant excited states have total spin $S=1$. We can also access specifically the lowest $S=1$ state by projector QMC simulations in the extended valence-bond basis with two unpaired spins, as we discuss in Sec. IVA 2. Previously, we used the mean length of the spinon strings in this basis 
(see Fig. 6) as a means to detect the AFM-RS transition. The strings can also provide spatial information in the form of the site-dependent string density, which for a string with $n$ sites in a given configuration (transition graph) is $\rho_{r}=1 / n$ for sites $r$ covered by the string and 0 otherwise [112]. Averaging over the simulation, we obtain the mean string density $\rho(x, y)$, which should reflect the spatial structure of the lowest $S=1$ wave function. A quantity similar to the string density was previously studied in a site-diluted Heisenberg model at the percolation point, and it gave useful information on the nature of quasilocalized moments in that case [97].

In the picture we outline for the RS state, the lowest $S=1$ state should be formed mainly from the localized $S=1 / 2$ spinons corresponding to breaking some of the singlets formed among groups of spinons. In a large system, the lowest excitation may not involve all the spinons, but in the relatively small systems we can access here, there is typically some string density in all regions identifiable as spinons. We expect a given string to be mainly confined to a region corresponding to a localized spinon, but the strings will also migrate between spinons (those involved significantly in the lowest $S=1$ state), and this should lead to elevated string density also on the domain walls. This migration of spinons should take place mainly within the same sublattice, i.e., a sublattice- $X$ spinon $X \in\{A, B\}$ will migrate predominantly between spinons located on the $X$ sublattice (with interesting violations of this rule to be discussed in Sec. VID). Fluctuations of the strings between nearby $A$ and $B$ spinon regions should also take place along domain walls connecting them, reflecting the anticipated role of the domain walls in mediating antiferromagnetic spinon-spinon interactions.

In order to have a clear example of the string density in regions of spinons and domain walls, we show a case of the random- $J$ model with a small number of spinons in Fig. 26. Here the domains are larger than in the previous random- $Q$ instance considered (Figs. 8 and 25), and in Fig. 26(a), one can clearly identify four cases of meeting points of four domain walls. Accordingly, in Fig. 26(b) there are four islands of high string density (where the periodic boundary conditions should be noted). The two upper islands have much higher integrated string density than the other two, indicating that the lowest excitation mainly corresponds to breaking up a singlet formed between those two spinons.

On the linear color scale in Fig. 26(b), one cannot easily detect any structure corresponding to the domain walls. When considering instead a logarithmic scale, Fig. 26(c), we can see channels formed in the regions corresponding to domain walls in Fig. 26(a), though these channels are clearly much more spread out than the domain walls visualized as in Fig. 26(a). Note again, however, that the representation in Fig. 26(a) is based on mean values in which the spatial fluctuations of the domain walls are not apparent. There are domain walls also within the completely dark regions, but those walls do not represent short paths connecting spinons. The spinon-spinon interactions
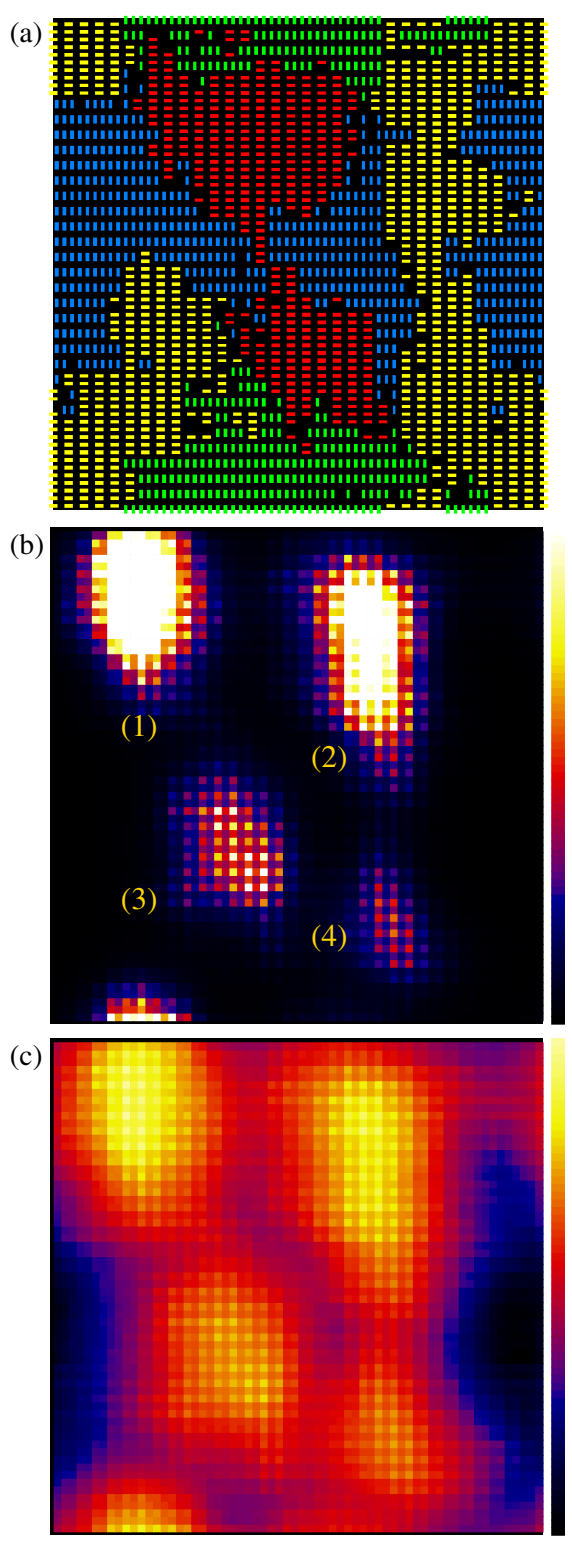

FIG. 26. Example of domain structure and spinon string density for an instance of the bimodal random- $J$ model. In (a), the bonds are color coded as in Fig. 8. The mean spinon string density $\rho(x, y)$ is graphed on a linear scale in (b), with the color bar corresponding to $\rho \in[0,0.002]$. The actual maximum value is $\rho \approx 0.017$, but all $\rho>0.002$ are shown as white in order to make the weaker features more visible. In (c), the color scale is for $\ln (\rho) \in[-15,-3]$ (the full range of the computed values). The labels 1-4 for the spinon regions in (b) are referenced in Fig. 28.

should be carried mainly along the shortest domain walls connecting spinons.

In Figs. 26(b) and 26(c), checkerboard patterns can be observed in the spinon regions. The pattern arises from the sublattice imbalance of the strings, which always cover an odd number of sites and the end points of which always stay on the same sublattice. Thus, the string density originating from an $X$-sublattice spinon $X \in\{A, B\}$ is higher on the $X$ sublattice. 


\section{Dynamic spinons}

The string density shown in Figs. 26(b) and 26(c) is the total density, with equal contributions from the $A$ and $B$ sublattice strings. Figure 27 shows the $A$ and $B$ densities separately. Here we can see clearly that the $A$ and $B$ strings are attracted predominantly, but not exclusively, to different regions of the lattice. The small but noticeable coexistence of the two spinon strings within the same regions requires dynamical aspects of the spinons that are not captured within the essentially static picture of the localized spinons that we have had in mind throughout the discussion of the results so far. In particular, the results in Fig. 27 imply that one cannot consider a region of high string density as occupied by either a spinon or an antispinon, but there is some degree of both spinon and antispinon in each vortex region.

As apparent from the basic picture of a spinon in Fig. 3, we can attach sublattice labels $A$ and $B$ to spinons and antispinons, respectively, as we have frequently done. Thus, a spinon can be characterized by two labels, the spin- $z$ component $\sigma \in \uparrow, \downarrow$ as well as the sublattice label $X \in$ $\{A, B\}$ (spinon, antispinon). If we consider two regions, 1 and 2 , in which spinons can exist, a singlet can be written as

$\Psi_{S}=a_{R}\left(\uparrow_{1 A} \downarrow_{2 B}-\downarrow_{1 A} \uparrow_{2 B}\right)+a_{C}\left(\uparrow_{1 B} \downarrow_{2 A}-\downarrow_{1 B} \uparrow_{2 A}\right)$,

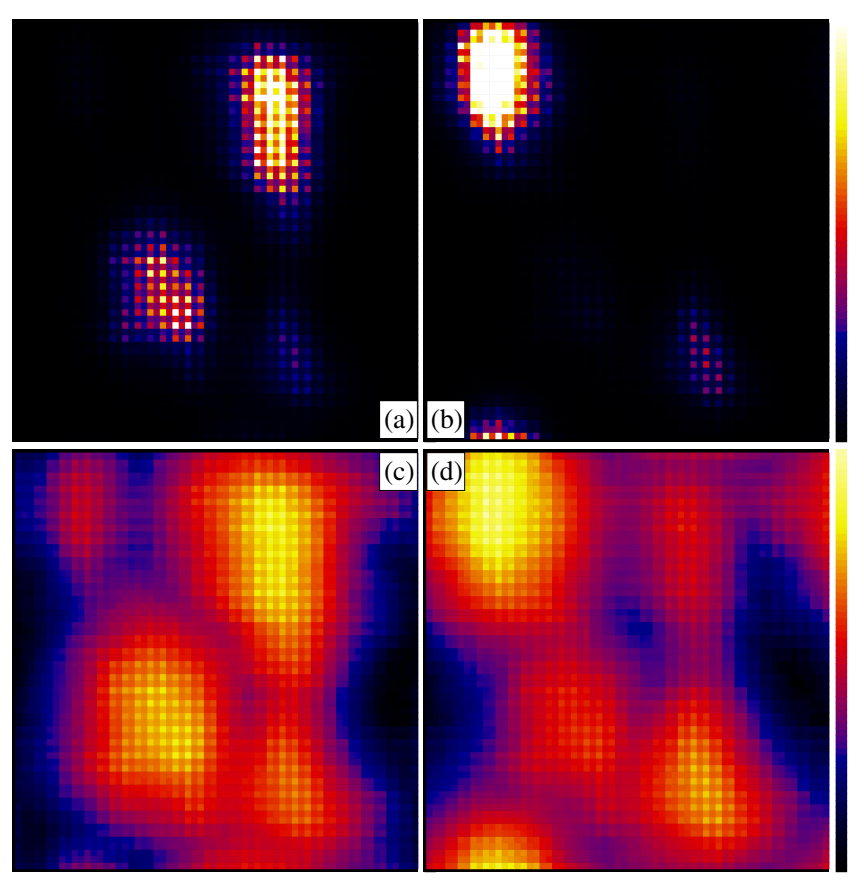

FIG. 27. The total string density $\rho$ in Fig. 26(b) resolved into contributions from the individual $A\left[\rho_{A}\right.$ in (a) and (c)] and $B\left[\rho_{B}\right.$ in (b) and (d)] strings. The scale is linear in (a) and (b), spanning the range [0, 0.003] (and white is used for larger values, up to 0.0097 for $\rho_{A}$ and 0.017 for $\rho_{B}$ ). In (c) and (d), the same data are shown on a logarithmic scale, with the color bar corresponding to $\ln \left(\rho_{A}\right), \ln \left(\rho_{B}\right) \in[-15,-3]$. where $\uparrow_{1 A}$ means that the spinon in region 1 is on sublattice $A$ with spin $\uparrow$, etc., and we demand that the two spinons cannot simultaneously occupy the same region. If region 1 is predominantly occupied by the spinon (i.e., it sits on sublattice $A$ ) and region 2 is occupied by the antispinon, then we consider the first term as the regular $(R)$ term and the second $(C)$ term is the cross term, and $\left|a_{R}\right| \gg\left|a_{C}\right|$. Naturally, it is the details of the disordered couplings that determine which of the terms is the regular one-essentially, as in the static spinon picture. The static description implies that $a_{C}=0$, but what we see in Fig. 27 is that cross terms in fact must exist, not only in the simple two-spinon system but also in systems with more than two spinons. Thus, it is not completely correct to say that disorder leads to localized spinons of type $A$ or $B$ as dictated by the VBS vortices and antivortices, but the physics is more complicated and involves quantum fluctuations that exchange not only the spins forming singlets but also the sublattice labels of the different spinon regions, with the constraint of a total-spin singlet and equal numbers of $A$ and $B$ labels.

In the simple picture of a spinon as a vortex in the VBS structure as drawn in Fig. 3, the unpaired spin is tied to the core of the VBS vortex. It seems unlikely that the VBS vortices in a disordered system can migrate substantially, since they are formed due to the local random environment. The unpaired spins, however, can migrate, and this is made easier owing to the presence of valence bonds longer than the shortest bonds in the simple picture in Fig. 3. The longer valence bonds also connect only spins on different sublattices, and in the case of a single VBS vortex on an infinite lattice, the unpaired spin cannot dissociate completely from the vortex core as long as there is VBS order and the probability of very long bonds decays exponentially with the bond length. Thus, an $X$-type spinon $X \in$ $\{A, B\}$ is the composite object of a VBS $X$-type vortex and an unpaired spin on sublattice $X$. However, in the case of a disorder-induced vortex-antivortex pair, there will be some probability of an unpaired spin on the $A$ sublattice to migrate to the antivortex associated with the $B$ sublattice, and vice versa. Thus, the unpaired spins are not completely tied to the VBS vortices on their own native sublattices. Here we consider the unpaired spins as the spinons (on sublattice $A$ ) and antispinons (on sublattice $B$ ) and refer to the VBS vortices and antivortices as separate objects. A spinon (antispinon) is still predominantly associated with a VBS vortex centered on sublattice $A$ (antivortex centered on sublattice $B$ ). We disregard the presumably low probability of a spinon and antispinon existing simultaneously at the same VBS vortex or antivortex.

It is important to note that there is no symmetry analogous to the $\mathrm{SU}(2)$ symmetry of the spins in the sublattice labels; instead, one should think of the spinons and antispinons as moving in different random potentials (likely with some repulsive interactions between spinons and antispinons in the same vortex region, though we do 


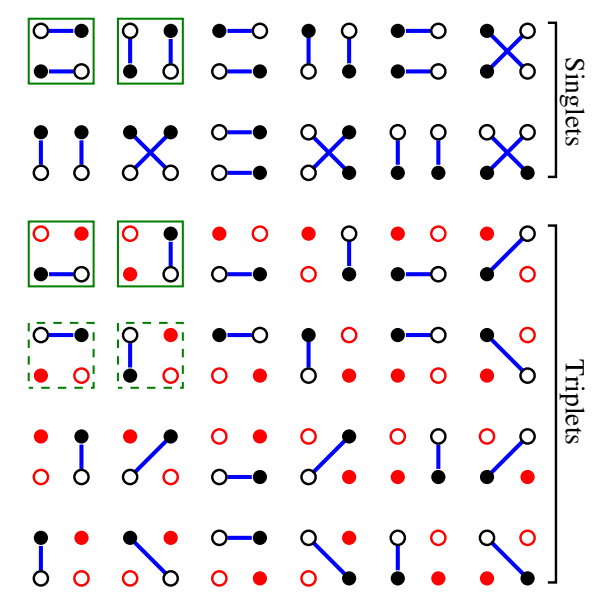

FIG. 28. Spinon wave-function components for singlet and triplets of two spinons and two antispinons, with the circles in order ${ }_{34}^{1} 2$ corresponding to the bright (spinon) regions in Fig. 26. Spinons (on sublattice $A$ ) and antispinons (on sublattice $B$ ) are denoted by solid and open circles, respectively, and singlet pairs are indicated by lines connecting circles. Unpaired red circles correspond to up spins (or other triplet states of the pairs). The components enclosed by solid green squares represent the dominant components of the wave functions as discussed in connection with Fig. 27, and the dashed green boxes indicate further regular components in the triplet sector. All other configurations correspond to cross terms existing when spinons and antispinons can trade places.

not quantify this). It is remarkable that these potentials, as is apparent in Fig. 27, both have minimums at roughly the same locations (the VBS vortices), instead of the spinons being repelled by the VBS antivortices and antispinons being repelled by the VBS vortices. This suggests a picture of the excited spinons as itinerant particles that can tunnel through channels corresponding to the domain walls between regions attracting both $A$ and $B$ spinons but with a typically large difference in the depth of the $A$ and $B$ potential wells ( $A$ and $B$ spinons being more attracted to type $A$ and $B$ VBS vortices, respectively). The excitations (of which we study only the lowest one here) should be localized in the sense that a given spinon migrates only substantially between a limited subset of the VBS vortices.

It is instructive to construct a general wave function for four spinons satisfying the above constraint and to analyze the $A$ and $B$ string densities in Fig. 27 within that formal framework. Figure 28 shows all possible singlet and triplet components of wave functions for two spinons and two antispinons. Here, the four circles are arranged to correspond closely to the four regions of elevated string density in Fig. 27 (which form roughly the corners of a square). The single dominant bright spot occupied by the $B$ string and the two bright spots (one of which is dominant) containing the $A$ string can be achieved in the triplet channel if the predominant wave-function components are the two enclosed by the solid-line boxes in Fig. 28. The dashed-line boxes enclose the two other triplet components corresponding to the same
$A, B$ arrangements but with the dimmer $A$-string spot in Fig. 27(b) having one of the unpaired spins instead of the brighter spot. Based on these triplet components, we can deduce that the dominant singlet components are those two indicated by squares. All other components, in both the singlet and the triplet sectors, are analogues of the cross term in the two-spinon state Eq. (22), and they have smaller amplitudes that can, in principle, be roughly estimated from the integrated densities within the different spots in Fig. 27. Doubly occupied vortices should also have some contributions in the wave function, but for simplicity we neglect those here.

It is not completely clear what the consequences are of these dynamical spinon effects. Most likely, the fluctuations are secondary effects, and the RS fixed point can be realized even with frozen $A, B$ labels. However, this hypothesis still needs to be tested. It is possible that the $A-B$ exchange processes actually contribute to the effective spinon-spinon interactions and, thus, further reinforce the scenario of spinon-antispinon singlet pairing as the mechanism responsible for the RS phase.

\section{CONCLUSIONS AND DISCUSSION}

\section{A. Summary}

Using the $J-Q$ model, we demonstrate that an RS phase can be induced by disorder in a quantum-spin system even though all microscopic interactions are bipartite, lacking the geometric frustration that so far was believed to be a necessary ingredient for this type of 2D state. The RS phase is characterized by algebraically decaying mean spin and dimer correlations, with distance dependence $\propto r^{-2}$ and $\propto r^{-4}$, respectively. For the continuous AFM-RS transition, we have sufficient evidence to conjecture universal critical exponents: $\nu=2, \eta=0$, and $z=2$ (with $\nu$ affected by the largest uncertainty). The $r^{-2}$ form of the spin correlation function also applies inside the RS phase, and this implies the exponent relation $\eta=2-z$ based on standard definitions of critical correlation functions [33]. The dynamic exponent $z$ increases from 2 as the RS phase is entered according to our results for a corresponding power-law divergent uniform and local magnetic susceptibilities. The observed consistency of the scaling forms for $\chi_{u}(T)$ and $\chi_{\mathrm{loc}}(T)$ [Eqs. (16) and (18)], with the latter derived under the condition of the exponent relation $\eta=2-z$ obtained at $T=0$, provides strong evidence for a critical RS phase in which the standard quantum-critical scaling laws apply.

The key physical mechanism underlying the RS phase argued in the case of the triangular lattice by Kimchi et al. [19] and observed directly here in our simulations of the square lattice is the pairwise creation of localized spinons (spinon-antispinon pairs) as the VBS is broken up into domains in the presence of disorder (which is similar also to the previously observed RS state arising out of the dimerized phase of the $J-Q$ chain [41]). This correlated 
spinon distribution leads to a network of weakly interacting spinon pairs and no long-range AFM order, in sharp contrast to the case of weak AFM order in systems with completely randomly distributed unfrustrated magnetic moments. We also argue that the VBS domain walls connecting spinons act as channels mediating the effective spinon-spinon interactions within the pairs. Moreover, we find a dynamical effect whereby the individual spatial regions containing the spinons cannot be associated purely with spinons or antispinons, but there is some mixing originating from migration of unpaired spins between the VBS vortices and antivortices. A given VBS vortex can still be classified either as predominantly a spinon or an antispinon according to the vortex type, and the mixing may not be necessary for describing the RS fixed point.

Because of the presence of spinons and their dominant role in the physical properties of the RS state, this state should not be referred to as a valence-bond glass, which is a term normally reserved for a state with random arrangements of short valence bonds, with critical dimer correlations but no liberated spinons [77] (though in the literature other kinds of states have also been referred to using the same term, e.g., in Refs. [13-15]). The spin correlations in the valence-bond glass were not discussed explicitly in Ref. [77], but they should decay exponentially in such a 2D state with only short valence bonds.

It is interesting to compare the critical exponents we obtain here at the AFM-RS transition with those at the transition between a superfluid and a Bose glass in the Bose-Hubbard model with random potentials [33]. Though the symmetries are different, the superfluid breaking $U(1)$ symmetry and the AFM state considered here breaking $O(3)$ symmetry, the exponents that we obtain here appear to be the same or satisfy the same bounds. At the Boseglass transition in $D$ dimensions, the dynamic exponent $z=D$, the same as $z=2=D$ that we find here. Also, the correlation length exponent $\nu=2$ at the Bose-glass transition, which we are not able to fully confirm in the case of the AFM-RS transition but is conjectured based on the results shown in Fig. 17. Moreover, in the Bose-glass case, the anomalous dimension should satisfy the bound $\eta \geq 0$. The mean spin correlations decaying as $1 / r^{2}$ in the RS phase and at the AFM-RS phase boundary corresponds to $\eta=0$ at the transition and the bound is satisfied. Apart from the obviously different symmetries, the BG phase is a Griffiths phase, which we argue is not the case for the RS phase. Therefore, the two transitions should not be expected to belong to the same universality class. The fact that the exponents nevertheless appear to be the same is intriguing and deserves further study.

It appears most likely that the RS state identified here is the same one, in the RG sense, as those previously conjectured in frustrated systems [13-20], though the lack of definitive quantitative results in the previous works (e.g., exponents governing various power-law behaviors) makes it difficult to definitely ascertain this at the moment. For example, it was argued that the low- $T$ susceptibility follows a Curie form in the frustrated honeycomb Heisenberg model in the RS phase [16], while we demonstrate here a $T^{-a}$ behavior with varying $a<1$ in the random $J-Q$ model (and $a \rightarrow 0$ as the AFM phase is approached). However, ED studies of lattices with only up to approximately 20 sites cannot be used to reliably address the detailed form of the divergence, as we see even with much larger systems here. In the work of Kimchi et al. [18] as well, it was not possible to obtain quantitative values of most of the exponents pertaining to the RS phase in the triangular lattice, though we note a more recent work in which scaling forms for the heat capacity (which we have not yet investigated) were obtained under various conditions and compared with experiments [19]. We note, in particular, that the previous works have not discussed any details of the AFM-RS phase transitions for which we obtain specific results here on power laws both at $T=0$ and $T>0$. In any case, there are no apparent contradictions between our RS state and that of Kimchi et al., and, given that the proposed mechanisms underlying the formation of these states are similar, a common RS fixed point appears plausible. We still discuss below our results in the context of other possible scenarios.

\section{B. Fixed points}

In the case of the triangular lattice, it was pointed out that the RS phase may eventually, at the longest length scales, be unstable to the formation of a spin-glass state [18]. Similarly, the square-lattice random $J-Q$ model might possibly be unstable to the formation of weak AFM order, though we see no signs of this up to the largest lattice studied here $(L=64)$. The fact that we observe such good scaling up to these system sizes at the very least implies that an RS fixed point exists (in the models studied here or outside but close to the present model space) and is responsible for the observed behaviors. The question then is whether there is one or two fixed points-one for bipartite interactions and one for frustrated interactions (perhaps above some critical strength of the frustration). We discuss possible RG scenarios for either case.

(i) There is a single RS fixed point. Let us call this the bipartite RS fixed point (BRSFP), even though it may attract also frustrated systems. A random system flowing to this fixed point has a true RS ground state. One possibility is that the BRSFP is stable for bipartite interactions but unstable when frustrated interactions are included; in that case, an interesting question is whether the fixed point is unstable in the presence of arbitrarily weak frustration or only above a critical frustration strength. Adapting the picture of Kimchi et al. [18] to this scenario, the flow away from the BRSFP would eventually lead to a spin-glass fixed point if the frustration is sufficiently strong [113]. Another possibility is that the BRSFP is also unstable in many 
bipartite systems (i.e., reaching it would require fine-tuning of parameters), and in that case the flow would be toward an AFM ordered fixed point. Even then, provided that the length scale at which the flow deviates from the BRSFP is sufficiently large, there will still be experimentally observable consequences of the proximity to the BRSFP fixed point (e.g., the temperature-dependent susceptibilities studied in Sec. V).

(ii) There are two fixed points: the BRSFP that we discuss above as well as a frustrated-RS fixed point (FRSFP). In this case, frustrated interactions, arbitrarily weak or above a critical strength, would cause a flow toward the FRSFP. These fixed points (both of them or only one of them) could also require fine-tuning, in principle. As in scenario (i), eventual flows away from the fixed points would still lead to experimentally observable consequences if the flows lead sufficiently close to the fixed points. If different, the BRSFP and FRSFP must have some differences in their operator contents, and there should then be some ways to distinguish them in numerical model studies and in experiments. As we mention above, so far there are no explicit indications of two fixed points based on existing numerics.

In addition to the several fixed points that we mention above - the BRSFP, FRSFP, AFM, and the spin glassthere could also be various other "random spin-liquid" fixed points in a wider space of disordered frustrated and bipartite quantum magnets. Experimentally, the question of how to distinguish between a spin glass and a random spin liquid has attracted considerable attention [21,114], and the issue is as of now unresolved. On general grounds, one would expect gapped (topological) spin liquids to be stable to weak disorder, while gapless (algebraic) spin liquids may generically flow to RS fixed points (either the BRSFP or the FRSFP).

In conjunction with the RS mechanism, we discuss the association between sublattices, VBS vortices, and spinons in Sec. VID. When frustrated interactions are added to a system on the square lattice, as long as the local VBS domains remain of the columnar type, the vortices and antivortices can still be uniquely associated with sublattices $A$ and $B$, even though the unpaired spins (the spinon strings discussed in Sec. VID) are not strictly confined to a given sublattice. However, as long as the frustration is not too strong, there will still be a sublattice preferred by the unpaired spin in a given vortex or antivortex in the same way as in the unfrustrated system, and the RS mechanism we discuss should remain valid. On other nonbipartite lattices, the division of sites into sublattices $A$ and $B$ is no longer possible, but there will still be both vortices and antivortices classified according to how the VBS angle changes when going around a vortex. An unpaired spin should also still be primarily associated with either a vortex or an antivortex. As long as the effective spinon-spinon interactions within a vortex-antivortex pair are antiferromagnetic, which has been argued to be the case by Kimchi et al. [18] in the case of the triangular lattice, the picture of dominantly short-distance pairing of the spinons (through their lattice correlations and interactions mediated by the domain walls) should apply. This mechanism would again suggest a common RS fixed point for frustrated and unfrustrated systems, though potentially strong frustration could invalidate the picture of local VBS domains and lead to a different state (e.g., a spin glass, as discussed by Kimchi et al. [18]).

If indeed the BRSFP encompasses the $J-Q$ model as well as the multitude of frustrated quantum magnets, the ability to study bipartite systems with large-scale unbiased QMC simulations has significant consequences in the context of experiments. It will then be possible to relate observed power laws directly to unbiased calculations, e.g., to test relationships between the power laws for different physical observables. Although the $J-Q$ model does not represent the correct microscopic interactions of specific materials, its phases can still contain the experimentally relevant low-energy physics. This is in the spirit of designer Hamiltonians [115], which are tailored to realize collective quantum states and quantum-phase transitions, while at the same time being amenable to numerical calculations, especially sign-free QMC simulations, on large scales without approximations. Given some of they key results that we obtain here, such as the $r^{-2}$ form of the decay of the mean spin correlation functions and the temperatureindependent magnetic susceptibility at the AFM-RS transition (and the divergent behavior with a varying exponent inside the RS phase), targeted calculations and scaling analysis aiming at these specific universal characteristics can hopefully soon be carried out also for the frustrated models. This would allow tests of our conjecture of a common RS fixed point for bipartite and frustrated systems.

One promising calculational route here is tensor network states tailored specifically to disordered spin models $[116,117]$. Though such calculations are certainly challenging, it may still be possible to reach larger system sizes than in the previous exact ED and DMRG studies. There has also been recent progress in improved DMRG methods for disordered systems [118], which may help in reaching larger system sizes. In the presence of weak frustration in the $J-Q$ model (or other relevant systems), it may be possible to obtain some QMC results even though the sign problem will limit the accessible system sizes and temperatures. In this context, it would also be useful to explore other bases in which the algorithms are formulated, with the goal of eliminating or strongly reducing the sign problem in some parameter regions, as in recent work on uniform frustrated quantum magnets [119-121].

A crucial question is whether and how the RS fixed point (s) can be obtained in SDRG calculations. The key physical ingredients underlying the RS phase-VBS domains and localized spinons - are unlikely generated correctly in the initial (high-energy) stages of the SDRG procedure applied 
directly to microscopic bipartite Heisenberg Hamiltonians; in $1 \mathrm{D}$, the method can only partially reproduce VBS domains [41]. It is furthermore very difficult to apply the SDRG approach to more complicated interactions like the six-spin $Q$ terms used here (which are difficult to deal with even in 1D systems [41]) since many kinds of effective couplings can be generated. It may be more fruitful to consider SDRG calculations carried out on a suitably constructed effective subsystem of the localized spinonantispinon pairs with their domain-wall-mediated interactions. With the $A-B$ sublattice correlation effect built into such an effective model, in combination with suitable inter- and intrapair coupling distributions, singlets should gradually freeze out one by one in a SDRG procedure. Like in $1 \mathrm{D}$, a rare-event mechanism [38,39] would likely be responsible for some pairing over larger distances, which is required for obtaining power-law correlations. It would be interesting to carry out SDRG calculations on effective models of randomly located spinons with different degrees of pair formation among $A$ and $B$ sublattice spins.

\section{Experiments}

A promising system for realizing a square-lattice RS state is the quasi-2D material $\mathrm{Sr}_{2} \mathrm{CuTe}_{1-x} \mathrm{~W}_{x} \mathrm{O}_{6}$, which was initially synthesized at $x=0,0.5$, and 1 [23], and more recently also for several other values of $x \in[0,1]$ [24,25]. The corresponding isostructural compounds $\mathrm{Sr}_{2} \mathrm{CuTeO}_{6}$ and $\mathrm{Sr}_{2} \mathrm{CuWO}_{6}$ have dominant nearest- and next-nearestneighbor spin interactions, respectively, owing to the different orbital properties of the plaquette centered Te and $\mathrm{W}$ ions. With random distribution of these ions, it was argued in Ref. [24] that an RS-type state forms in a sizable region of $0<x<1$, though detailed comparisons with specific RS predictions were still lacking. In Ref. [25], it was instead argued that the state of the random system is a valence-bond glass with a singlet gap. Within our scenario, the RS state on the square lattice could form as a consequence of couplings locally favoring VBS domains, and this could possibly be the case when the $J_{1}$ and $J_{2}$ couplings are mixed at random, even though the pure $J_{1}$ and $J_{2}$ systems are magnetically ordered (with Néel and stripe AFM order for $J_{1}$ and $J_{2}$ couplings, respectively). Note that the uniform frustrated Heisenberg model with variable $J_{2} / J_{1}$ has a VBS phase in its phase diagram [68-70].

An intriguing observation $[24,25]$ is a divergent low- $T$ susceptibility for $x \in[0.2,0.5]$. This divergence was interpreted as a Curie tail originating from isolated magnetic moments in the random systems. In light of the findings we present here for the susceptibility in the RS state, we reanalyze the susceptibility data of Ref. [25] (Fig. 2) in the regime of Te-W mixing $x$ where RS physics may pertain. Figure 29 shows the low-temperature susceptibility for the $\mathrm{W}$ fraction $x$ in the range $0.2-0.5$ fitted to the form $\chi_{u}=\chi_{0}+c T^{-a}$. We use two different temperature windows for these fits, $T<4 \mathrm{~K}$ (shown as red curves) and $T<3 \mathrm{~K}$ (blue curves). In all cases,
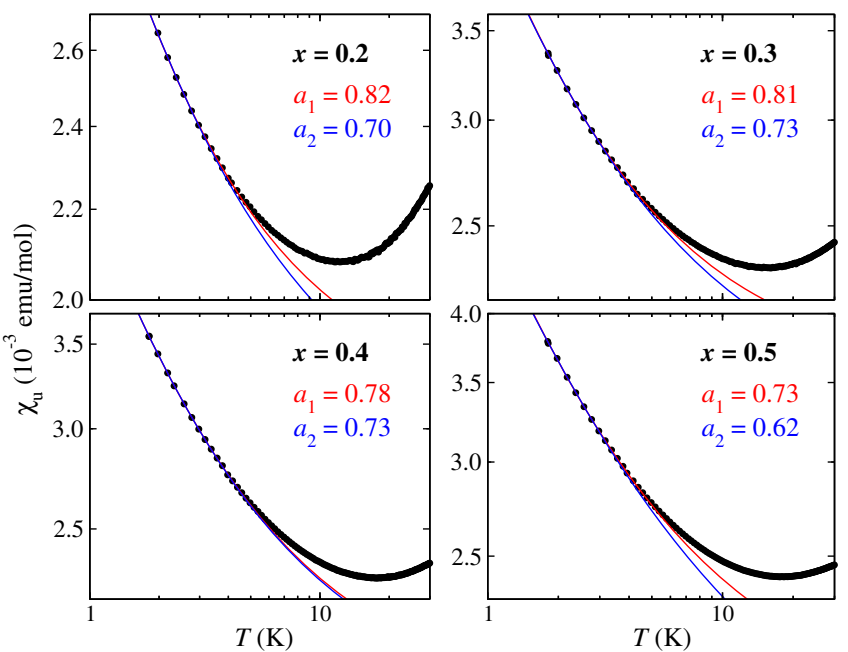

FIG. 29. Experimental susceptibility data (black circles) for the random quantum magnet $\mathrm{Sr}_{2} \mathrm{CuTe}_{1-x} \mathrm{~W}_{x} \mathrm{O}_{6}$ from Ref. [25]. The panels correspond to $\mathrm{W}$ fractions $x=0.2,0.3,0.4,0.5$, as indicated. Logarithmic scales are used for all axes. The curves are of the form $\chi_{u}=\chi_{0}+c T^{-a}$, with red and blue corresponding, respectively, to $T<4 \mathrm{~K}$ and $T<3 \mathrm{~K}$ data used in the fits. The temperature exponents ( $a_{1}$ for $T<4 \mathrm{~K}$ and $a_{2}$ for $T<3 \mathrm{~K}$ ) obtained from the fits are also indicated in the panels.

we find that the divergence is slower than the Curie law. For the $T<4 \mathrm{~K}$ fits, we obtain exponents $a$ in the range $0.73-0.82$, while the range is $0.62-0.73$ when the lower cutoff is used. An important observation is that the exponent consistently decreases when the temperature cutoff is reduced. This makes it seem unlikely that the low- $T$ susceptibility follows the Curie law, though we note that reasonable fits can also be obtained with $a=1$; these fits work approximately up to considerably higher temperatures than those shown in Fig. 29, but the low- $T$ data are not as closely matched as with the fits in Fig. 29.

While these data fits are not conclusive, the findings motivate further experimental studies and analysis based on the concrete RS predictions we report here. Experiments at still lower temperatures would be desirable in this regard. It would be particularly interesting to test our prediction of a temperature-independent low- $T$ susceptibility at the AFM-RS transition.

\section{Future extensions}

Many interesting QMC calculations are called for as extensions of the initial study of the random $J-Q$ model presented here. For example, the evolution of the RS phase as a function of an external magnetic field (which was recently studied in the triangular lattice [19]) is very interesting theoretically and also from the experimental perspective. The field can be included in SSE simulations of the $J-Q$ model $[122,123]$. Dynamical signatures, e.g., the dynamic spin-structure factor, can also be studied using SSE supplemented by analytic continuation techniques [124], 
and it will be interesting to compare the 2D RS phase with the random-exchange Heisenberg chain, which was also recently studied with the abovementioned techniques [125]. We also note that the specific heat played a major role in the experiments in Ref. [25] and also theoretically in the context of other materials in Ref. [19]. We do not report specific heat QMC results here because they require significantly more computational resources than the susceptibility. We plan to calculate the specific heat in future work.

The diluted $J-Q$ model also deserves further studies. Here we merely confirm that it does not have an RS ground state but hosts weak AFM order. However, the system mixes aspects of vacancy-induced moments and RS physics, and potentially, it could exhibit clear RS behaviors on intermediate length scales; e.g., it may show an anomalous divergent susceptibility similar to that of a system with RS ground state. This kind of behavior was indeed suggested recently in the context of random spin liquids in frustrated quantum magnets, where experimentally one may expect the presence of more than one type of impurity moment, including localized spinons, and these different interacting moments may collectively cause a non-Curie susceptibility [126]. Given the similarities we discuss here between the $Q$ terms and frustrated interactions, it would be interesting to investigate the susceptibility and other experimentally relevant $T>0$ properties of the diluted $J-Q$ model. Other variants of the $J-Q$ model can also be studied in order to test the universality of the RS-AFM transition and the RS state, e.g., the $J-Q_{2}$ model with two instead of three singlet projectors in Eq. (1) and Fig. 1 [127].

\section{ACKNOWLEDGMENTS}

We would like to thank K. Beach, I. Kimchi, T. Okubo, S. Sachdev, T. Senthil, J. Takahashi, and L. Wang for valuable discussions. We are grateful to the authors of Ref. [25] for sending us the experimental susceptibility data displayed in Fig. 29. W. G. is supported by NSFC under Grants No. 11734002 and No. 11775021. H. S. is supported by the China Postdoctoral Science Foundation under Grants No. 2016M600034 and No. 2017T100031 and by Boston University's Condensed Matter Theory Visitors Program. Y. -C. L. is supported by the MOST (Taiwan) and the National Center for Theoretical Sciences (Taiwan). A. W. S. is supported by the NSF under Grant No. DMR1710170 and by a Simons Investigator Award, and he also gratefully acknowledges travel support from Beijing Normal University under YinZhi Project No. C2018046, as well as from the National Center for Theoretical Sciences. Some of the numerical calculations are carried out on Boston University's Shared Computing Cluster.

[1] R. Nandkishore and D. A. Huse, Many-Body Localization and Thermalization in Quantum Statistical Mechanics, Annu. Rev. Condens. Matter Phys. 6, 15 (2015).
[2] J. Smith, A. Lee, P. Richerme, B. Neyenhuis, P. W. Hess, P. Hauke, M. Heyl, D. A. Huse, and C. Monroe, Many-Body Localization in a Quantum Simulator with Programmable Random Disorder, Nat. Phys. 12, 907 (2016).

[3] N. Y. Yao, L. Jiang, A. V. Gorshkov, Z.-X. Gong, A. Zhai, L.-M. Duan, and M. D. Lukin, Robust Quantum State Transfer in Random Unpolarized Spin Chains, Phys. Rev. Lett. 106, 040505 (2011).

[4] Y. Shimizu, K. Miyagawa, K. Kanoda, M. Maesato, and G. Saito, Spin Liquid State in an Organic Mott Insulator with a Triangular Lattice, Phys. Rev. Lett. 91, 107001 (2003).

[5] S. Yamashita, Y. Nakazawa, M. Oguni, Y. Oshima, H. Nojiri, Y. Shimizu, K. Miyagawa, and K. Kanoda, Thermodynamic Properties of a Spin-1/2 Spin-Liquid State in a $\kappa$-Type Organic Salt, Nat. Phys. 4, 459 (2008).

[6] R. S. Manna, M. de Souza, A. Brühl, J. A. Schlueter, and M. Lang, Lattice Effects and Entropy Release at the LowTemperature Phase Transition in the Spin-Liquid Candidate $\kappa$-(BEDT-TTF $)_{2} \mathrm{Cu}_{2}(\mathrm{CN})_{3}$, Phys. Rev. Lett. 104, 016403 (2010).

[7] F. L. Pratt, P. J. Baker, S. J. Blundell, T. Lancaster, S. Ohira-Kawamura, C. Baines, Y. Shimizu, K. Kanoda, I. Watanabe, and G. Saito, Magnetic and Non-Magnetic Phases of a Quantum Spin Liquid, Nature (London) 471, 612 (2011).

[8] S.-H. Lee, H. Kikuchi, Y. Qiu, B. Lake, Q. Huang, K. Habicht, and K. Kiefer, Quantum-Spin-Liquid States in the Two-Dimensional Kagome Antiferromagnets $\mathrm{Zn}_{x} \mathrm{Cu}_{4-x}(\mathrm{OD})_{6} \mathrm{Cl}_{2}$, Nat. Mater. 6, 853 (2007).

[9] M. A. de Vries, J. R. Stewart, P. P. Deen, J. O. Piatek, G. J. Nilsen, H. M. Rönnow, and A. Harrison, Scale-Free Antiferromagnetic Fluctuations in the $s=1 / 2$ Kagome Antiferromagnet Herbertsmithite, Phys. Rev. Lett. 103, 237201 (2009).

[10] J. S. Helton, K. Matan, M. P. Shores, E. A. Nytko, B. M. Bartlett, Y. Qiu, D. G. Nocera, and Y. S. Lee, Dynamic Scaling in the Susceptibility of the Spin-1/2 Kagome Lattice Antiferromagnet Herbertsmithite, Phys. Rev. Lett. 104, 147201 (2010).

[11] T.-H. Han, J. S. Helton, S. Chu, D. G. Nocera, J. A. Rodriguez-Rivera, C. Broholm, and Y. S. Lee, Fractionalized Excitations in the Spin-Liquid State of a KagomeLattice Antiferromagnet, Nature (London) 492, 406 (2012).

[12] R. R. P. Singh, Valence Bond Glass Phase in Dilute Kagome Antiferromagnets, Phys. Rev. Lett. 104, 177203 (2010).

[13] K. Watanabe, H. Kawamura, H. Nakano, and T. Sakai, Quantum Spin-Liquid Behavior in the Spin-1/2 Random Heisenberg Antiferromagnet on the Triangular Lattice, J. Phys. Soc. Jpn. 83, 034714 (2014).

[14] H. Kawamura, K. Watanabe, and T. Shimokawa, Quantum Spin-Liquid Behavior in the Spin-1/2 Random-Bond Heisenberg Antiferromagnet on the Kagome Lattice, J. Phys. Soc. Jpn. 83, 103704 (2014).

[15] T. Shimokawa, K. Watanabe, and H. Kawamura, Static and Dynamical Spin Correlations of the $S=1 / 2$ RandomBond Antiferromagnetic Heisenberg Model on the Triangular and Kagome Lattices, Phys. Rev. B 92, 134407 (2015). 
[16] K. Uematsu and H. Kawamura, Randomness-Induced Quantum Spin Liquid Behavior in the $s=1 / 2$ Random $J_{1}-J_{2}$ Heisenberg Antiferromagnet on the Honeycomb Lattice, J. Phys. Soc. Jpn. 86, 044704 (2017);

[17] K. Uematsu and H. Kawamura, Randomness-Induced Quantum Spin Liquid Behavior in the $s=1 / 2$ Random $J_{1}-J_{2}$ Heisenberg Antiferromagnet on the Square Lattice, Phys. Rev. B 98, 134427 (2018).

[18] I. Kimchi, A. Nahum, and T. Senthil, Valence Bonds in Random Quantum Magnets: Theory and Application to $\mathrm{YbMgGaO}_{4}$, Phys. Rev. X 8, 031028 (2018).

[19] I. Kimchi, J. P. Sheckelton, T. M. McQueen, and P. A. Lee, Scaling and Data Collapse from Local Moments in Frustrated Disordered Quantum Spin Systems, Nat. Commun. 9, 4367 (2018).

[20] H.-Q. Wu, S.-S. Gong, and D. N. Sheng, Randomness Induced Spin-Liquid-like Phase in the Spin-1/2 $J_{1}-J_{2}$ Triangular Heisenberg Model, arXiv:1802.00935.

[21] Y. Li, G. Chen, W. Tong, L. Pi, J. Liu, Z. Yang, X. Wang, and Q. Zhang, Rare-Earth Triangular Lattice Spin Liquid: A Single-Crystal Study of $\mathrm{YbMgGaO}_{4}$, Phys. Rev. Lett. 115, 167203 (2015).

[22] Y. Li, D. Adroja, R. I. Bewley, D. Voneshen, A. A. Tsirlin, P. Gegenwart, and Q. Zhang, Crystalline Electric-Field Randomness in the Triangular Lattice Spin-Liquid $\mathrm{YbMgGaO}_{4}$, Phys. Rev. Lett. 118, 107202 (2017).

[23] O. Mustonen, S. Vasala, E. Sadrollahi, K. P. Schmidt, C. Baines, H. C. Walker, I. Terasaki, F. J. Litterst, E. Baggio-Saitovitch, and M. Karppinen, Spin-Liquid-like State in a Spin-1/2 Square-Lattice Antiferromagnet Perovskite Induced by $d^{10}-d^{0}$ Cation Mixing, Nat. Commun. 9, 1085 (2018).

[24] O. Mustonen, S. Vasala, K. P. Schmidt, E. Sadrollahi, H. C. Walker, I. Terasaki, F. J. Litterst, E. Baggio-Saitovitch, and M. Karppinen, Tuning the $S=1 / 2$ Square-Lattice Antiferromagnet $\mathrm{Sr}_{2} \mathrm{Cu}\left(\mathrm{Te}_{1-x} \mathrm{~W}_{x}\right) \mathrm{O}_{6}$ from Néel Order to Quantum Disorder to Columnar Order, Phys. Rev. B 98, 064411 (2018).

[25] M. Watanabe, N. Kurita, H. Tanaka, W. Ueno, K. Matsui, and T. Goto, Valence-Bond-Glass State with Singlet Gap in the Spin-1/2 Square-Lattice Random $J_{1}-J_{2}$ Heisenberg Antiferromagnet $\mathrm{Sr}_{2} \mathrm{CuTe}_{1-x} \mathrm{~W}_{x} \mathrm{O}_{6}$, Phys. Rev. B 98, 054422 (2018).

[26] M. Levin and T. Senthil, Deconfined Quantum Criticality and Néel Order via Dimer Disorder, Phys. Rev. B 70, 220403 (2004).

[27] T. Vojta, Quantum Griffiths Effects and Smeared Phase Transitions in Metals: Theory and Experiment, J. Low Temp. Phys. 161, 299 (2010).

[28] T. Vojta, Phases and Phase Transitions in Disordered Quantum Systems, AIP Conf. Proc. 1550, 188 (2013).

[29] N. Read, S. Sachdev, and J. Ye, Landau Theory of Quantum Spin Glasses of Rotors and Ising Spins, Phys. Rev. B 52, 384 (1995).

[30] M. Guo, R. N. Bhatt, and D. A. Huse, Quantum Critical Behavior of a Three-Dimensional Ising Spin Glass in a Transverse Magnetic Field, Phys. Rev. Lett. 72, 4137 (1994).

[31] M. Guo, R. N. Bhatt, and D. A. Huse, Quantum Griffiths Singularities in the Transverse-Field Ising Spin Glass, Phys. Rev. B 54, 3336 (1996).
[32] H. Rieger and A. P. Young, Griffiths Singularities in the Disordered Phase of a Quantum Ising Spin Glass, Phys. Rev. B 54, 3328 (1996).

[33] M. P. A. Fisher, P. B. Weichman, G. Grinstein, and D. S. Fisher, Boson Localization and the Superfluid-Insulator Transition, Phys. Rev. B 40, 546 (1989).

[34] S. Iyer, D. Pekker, and G. Refael, Mott Glass to Superfluid Transition for Random Bosons in Two Dimensions, Phys. Rev. B 85, 094202 (2012).

[35] R. Mélin, Antiferromagnetism in a Doped Spin-Peierls Model: Classical and Quantum Behaviors, Eur. Phys. J. B 16, 261 (2000).

[36] Y.-C. Lin, R. Mélin, H. Rieger, and F. Iglói, Low-Energy Fixed Points of Random Heisenberg Models, Phys. Rev. B 68, 024424 (2003).

[37] Y.-C. Lin, H. Rieger, N. Laflorencie, and F. Iglói, StrongDisorder Renormalization Group Study of $S=1 / 2 \mathrm{Hei}$ senberg Antiferromagnet Layers and Bilayers with Bond Randomness, Site Dilution, and Dimer Dilution, Phys. Rev. B 74, 024427 (2006).

[38] C. Dasgupta and S.-K. Ma, Low-Temperature Properties of the Random Heisenberg Antiferromagnetic Chain, Phys. Rev. B 22, 1305 (1980).

[39] D. S. Fisher, Random Antiferromagnetic Quantum Spin Chains, Phys. Rev. B 50, 3799 (1994).

[40] J. A. Hoyos and T. Vojta, Theory of Smeared Quantum Phase Transitions, Phys. Rev. Lett. 100, 240601 (2008).

[41] Y.-R. Shu, D.-X. Yao, C.-W. Ke, Y.-C. Lin, and A. W. Sandvik, Properties of the Random-Singlet Phase: From the Disordered Heisenberg Chain to an Amorphous Valence-Bond Solid, Phys. Rev. B 94, 174442 (2016).

[42] G. Refael and J.E. Moore, Entanglement Entropy of Random Quantum Critical Points in One Dimension, Phys. Rev. Lett. 93, 260602 (2004).

[43] R. Mélin, Y.-C. Lin, P. Lajkó, H. Rieger, and F. Iglói, Strongly Disordered Spin Ladders, Phys. Rev. B 65 , 104415 (2002).

[44] G. Refael, S. Kehrein, and D. S. Fisher, Spin Reduction Transition in Spin-3/2 Random Heisenberg Chains, Phys. Rev. B 66, 060402(R) (2002).

[45] S. Pielawa and E. Altman, Numerical Evidence for Strong Randomness Scaling at a Superfluid-Insulator Transition of One-Dimensional Bosons, Phys. Rev. B 88, 224201 (2013).

[46] R. N. Bhatt and P. A. Lee, Scaling Studies of Highly Disordered Spin-1/2 Antiferromagnetic Systems, Phys. Rev. Lett. 48, 344 (1982).

[47] C. Pich, A.P. Young, H. Rieger, and N. Kawashima, Critical Behavior and Griffiths-McCoy Singularities in the Two-Dimensional Random Quantum Ising Ferromagnet, Phys. Rev. Lett. 81, 5916 (1998).

[48] O. Motrunich, S.-C. Mau, D. A. Huse, and D. S. Fisher, Infinite-Randomness Quantum Ising Critical Fixed Points, Phys. Rev. B 61, 1160 (2000).

[49] R. Juhász, Y.-C. Lin, and F. Iglói, Strong Griffiths Singularities in Random Systems and Their Relation to Extreme Value Statistics, Phys. Rev. B 73, 224206 (2006). 
[50] Y.-C. Lin, F. Iglói, and H. Rieger, Entanglement Entropy at Infinite-Randomness Fixed Points in Higher Dimensions, Phys. Rev. Lett. 99, 147202 (2007).

[51] C. R. Laumann, D. A. Huse, A. W. W. Ludwig, G. Refael, S. Trebst, and M. Troyer, Strong-Disorder Renormalization for Interacting Non-Abelian Anyon Systems in Two Dimensions, Phys. Rev. B 85, 224201 (2012).

[52] F. Iglói and C. Monthus, Strong Disorder RG Approach of Random Systems, Phys. Rep. 412, 277 (2005).

[53] Y. Xing et al., Quantum Griffiths Singularity of Superconductor-Metal Transition in Ga Thin Films, Science 350, 542 (2015).

[54] N. Laflorencie, S. Wessel, A. Läuchli, and H. Rieger, Random-Exchange Quantum Heisenberg Antiferromagnets on a Square Lattice, Phys. Rev. B 73, 060403(R) (2006).

[55] J. Lou, A. W. Sandvik, and N. Kawashima, Antiferromagnetic to Valence-Bond-Solid Transitions in TwoDimensional $S U(N)$ Heisenberg Models with Multispin Interactions, Phys. Rev. B 80, 180414(R) (2009).

[56] A. W. Sandvik, Finite-Size Scaling and Boundary Effects in Two-Dimensional Valence-Bond Solids, Phys. Rev. B 85, 134407 (2012).

[57] E. Manousakis, The Spin-1/2 Heisenberg Antiferromagnet on a Square Lattice and Its Application to the Cuprous Oxides, Rev. Mod. Phys. 63, 1 (1991).

[58] A. W. Sandvik, Computational Studies of Quantum Spin Systems, AIP Conf. Proc. 1297, 135 (2010).

[59] A. W. Sandvik, Evidence for Deconfined Quantum Criticality in a Two-Dimensional Heisenberg Model with FourSpin Interactions, Phys. Rev. Lett. 98, 227202 (2007).

[60] R. G. Melko and R. K. Kaul, Scaling in the Fan of an Unconventional Quantum Critical Point, Phys. Rev. Lett. 100, 017203 (2008).

[61] F. J. Jiang, M. Nyfeler, S. Chandrasekharan, and U.-J. Wiese, From an Antiferromagnet to a Valence Bond Solid: Evidence for a First-Order Phase Transition, J. Stat. Mech. (2008) P02009.

[62] A. W. Sandvik, Continuous Quantum Phase Transition between an Antiferromagnet and a Valence-Bond Solid in Two Dimensions: Evidence for Logarithmic Corrections to Scaling, Phys. Rev. Lett. 104, 177201 (2010).

[63] K. Harada, T. Suzuki, T. Okubo, H. Matsuo, J. Lou, H. Watanabe, S. Todo, and N. Kawashima, Possibility of Deconfined Criticality in $S U(N)$ Heisenberg Models at Small N, Phys. Rev. B 88, 220408 (2013).

[64] K. Chen, Y. Huang, Y. Deng, A. B. Kuklov, N. V. Prokofev, and B. V. Svistunov, Deconfined Criticality Flow in the Heisenberg Model with Ring-Exchange Interactions, Phys. Rev. Lett. 110, 185701 (2013).

[65] M. S. Block, R. G. Melko, and R. K. Kaul, Fate of $C P^{N-1}$ Fixed Points with q Monopoles, Phys. Rev. Lett. 111, 137202 (2013).

[66] S. Pujari, F. Alet, and K. Damle, Transitions to ValenceBond Solid Order in a Honeycomb Lattice Antiferromagnet, Phys. Rev. B 91, 104411 (2015).

[67] W.-J. Hu, F. Becca, A. Parola, and S. Sorella, Direct Evidence for a Gapless $Z_{2}$ Spin Liquid by Frustrating Néel Antiferromagnetism, Phys. Rev. B 88, 060402(R) (2013).
[68] S. Morita, R. Kaneko, and M. Imada, Quantum Spin Liquid in Spin 1/2 $J_{1}-J_{2}$ Heisenberg Model on Square Lattice: Many-Variable Variational Monte Carlo Study Combined with Quantum-Number Projections, J. Phys. Soc. Jpn. 84, 024720 (2015).

[69] S.-S. Gong, W. Zhu, D. N. Sheng, O. I. Motrunich, and M. P. A. Fisher, Plaquette Ordered Phase and Quantum Phase Diagram in the Spin-1/2 $J_{1}-J_{2}$ Square Heisenberg Model, Phys. Rev. Lett. 113, 027201 (2014).

[70] L. Wang and A. W. Sandvik, Critical Level Crossings and Gapless Spin Liquid in the Square-Lattice Spin-1/2 $J_{1}-J_{2}$ Heisenberg Antiferromagnet, Phys. Rev. Lett. 121, 107202 (2018).

[71] L. Wang, Z.-C. Gu, F. Verstraete, and X.-G. Wen, TensorProduct State Approach to Spin-1/2 Square $J_{1}-J_{2}$ Antiferromagnetic Heisenberg Model: Evidence for Deconfined Quantum Criticality, Phys. Rev. B 94, 075143 (2016).

[72] R. Haghshenas and D. N. Sheng, U(1)-Symmetric Infinite Projected Entangled-Pair State Study of the Spin-1/2 Square $J_{1}-J_{2}$ Heisenberg Model, Phys. Rev. B 97, 174408 (2018).

[73] H. Shao, W. Guo, and A. W. Sandvik, Quantum Criticality with Two Length Scales, Science 352, 213 (2016).

[74] T. Senthil, A. Vishwanath, L. Balents, S. Sachdev, and M. P. A. Fisher, Deconfined Quantum Critical Points, Science 303, 1490 (2004).

[75] T. Senthil, L. Balents, S. Sachdev, A. Vishwanath, and M. P. A. Fisher, Quantum Criticality beyond the LandauGinzburg-Wilson Paradigm, Phys. Rev. B 70, 144407 (2004).

[76] Y. Imry and S. K. Ma, Random-Field Instability of the Ordered State of Continuous Symmetry, Phys. Rev. Lett. 35, 1399 (1975).

[77] M. Tarzia and G. Biroli, The Valence Bond Glass Phase, Europhys. Lett. 82, 67008 (2008).

[78] M. A. de Vries, A. C. Mclaughlin, and J.-W. G. Bos, Valence Bond Glass on an fcc Lattice in the Double Perovskite $\mathrm{Ba}_{2} \mathrm{YMoO}_{6}$, Phys. Rev. Lett. 104, 177202 (2010).

[79] J. P. Carlo, J. P. Clancy, T. Aharen, Z. Yamani, J. P. C. Ruff, J. J. Wagman, G. J. Van Gastel, H. M. L. Noad, G. E. Granroth, J. E. Greedan, H. A. Dabkowska, and B. D. Gaulin, Triplet and In-Gap Magnetic States in the Ground State of the Quantum Frustrated fcc Antiferromagnet $\mathrm{Ba}_{2} \mathrm{YMoO}_{6}$, Phys. Rev. B 84, 100404(R) (2011).

[80] A. Lavarélo and G. Roux, Localization of Spinons in Random Majumdar-Ghosh Chains, Phys. Rev. Lett. 110, 087204 (2013).

[81] N. Nagaosa, A. Furusaki, M. Sigrist, and H. Fukuyama, Nonmagnetic Impurities in Spin Gap Systems, J. Phys. Soc. Jpn. 65, 3724 (1996).

[82] R. R. P. Singh, M. P. Gelfand, and D. A. Huse, Ground States of Low-Dimensional Quantum Antiferromagnets, Phys. Rev. Lett. 61, 2484 (1988).

[83] M. Matsumoto, C. Yasuda, S. Todo, and H. Takayama, Ground-State Phase Diagram of Quantum Heisenberg Antiferromagnets on the Anisotropic Dimerized Square Lattice, Phys. Rev. B 65, 014407 (2001). 
[84] C. Yasuda, S. Todo, M. Matsumoto, and H. Takayama, Site-Dilution-Induced Antiferromagnetic Long-Range Order in a Two-Dimensional Spin-Gapped Heisenberg Antiferromagnet, Phys. Rev. B 64, 092405 (2001).

[85] T. Mendes-Santos, N. C. Costa, G. Batrouni, N. Curro, R. R. dos Santos, T. Paiva, and R. T. Scalettar, Impurities near an Antiferromagnetic-Singlet Quantum Critical Point, Phys. Rev. B 95, 054419 (2017).

[86] A. W. Sandvik, Ground State Projection of Quantum Spin Systems in the Valence-Bond Basis, Phys. Rev. Lett. 95, 207203 (2005).

[87] A. W. Sandvik and H. G. Evertz, Loop Updates for Variational and Projector Quantum Monte Carlo Simulations in the Valence-Bond Basis, Phys. Rev. B 82, 024407 (2010).

[88] A. W. Sandvik, Stochastic Series Expansion Method with Operator-Loop Update, Phys. Rev. B 59, R14157(R) (1999).

[89] H. Shao, W. Guo, and A. W. Sandvik, Emergent Topological Excitations in a Two-Dimensional Quantum Spin System, Phys. Rev. B 91, 094426 (2015).

[90] R. K. Kaul, R. G. Melko, M. A. Metlitski, and S. Sachdev, Imaging Bond Order near Nonmagnetic Impurities in Square-Lattice Antiferromagnets, Phys. Rev. Lett. 101, 187206 (2008).

[91] In a frustrated system, nonbipartite bonds, if present, do not vanish upon time evolution, but it should be noted that the bipartite valence-bond basis is always (over)complete; thus, on the square lattice one can also use a basis without frustrated bonds even when frustrated interactions are present. However, it is only when the interactions are bipartite that the ground state can be expressed as positivedefinite superpositions of valence-bond configurations.

[92] T. Sulejmanpasic, H. Shao, A. W. Sandvik, and M. Ünsal, Confinement in the Bulk, Deconfinement on the Wall: Infrared Equivalence between Compactified QCD and Quantum Magnets, Phys. Rev. Lett. 119, 091601 (2017).

[93] S. Liang, B. Doucot, and P. W. Anderson, Some New Variational Resonating-Valence-Bond-Type Wave Functions for the Spin-1/2 Antiferromagnetic Heisenberg Model on a Square Lattice, Phys. Rev. Lett. 61, 365 (1988).

[94] B. Sutherland, Systems with Resonating-Valence-Bond Ground States: Correlations and Excitations, Phys. Rev. B 37, 3786 (1988).

[95] K. S. D. Beach and A. W. Sandvik, Some Formal Results for the Valence Bond Basis, Nucl. Phys. B750, 142 (2006).

[96] A sign-free QMC simulation corresponds to a related classical statistical-mechanics problem, the properties of which reflect those of the quantum system of interest. Any phase transition taking place in the classical system must also correspond to a phase transition in the quantum system and vice versa. Thus, for the purpose of locating a phase transition in the quantum system, one does not necessarily have to study a property with a strict relationship to a true quantum-mechanical expectation value, but one can choose some quantity that is convenient to evaluate in the simulation. As an example, in valence-bond projector QMC simulations, $\left\langle D^{n}\right\rangle$ for $n>2$ is difficult to evaluate as an exact quantum expectation value, but by examining the relevant expressions [95], one can see that the finite-size scaling, at least to leading order, should be the same for $\left\langle(\tilde{D})^{n}\right\rangle$, where $\tilde{D}$ is the easily computed real number corresponding to the operator $D$ in a QMC transition graph [and $(\tilde{D})^{n}$ differs only slightly from the number $\tilde{D}^{n}$ giving the exact $\left\langle D^{n}\right\rangle$ ]. This replacement of the operator $D$ by the number $\tilde{D}$ is used when defining the Binder cumulant of the dimer order parameter.

[97] L. Wang and A. W. Sandvik, Low-Energy Excitations of Two-Dimensional Diluted Heisenberg Quantum Antiferromagnets, Phys. Rev. B 81, 054417 (2010).

[98] Y. Tang and A. W. Sandvik, Method to Characterize Spinons as Emergent Elementary Particles, Phys. Rev. Lett. 107, 157201 (2011).

[99] D. S. Fisher and A. P. Young, Distributions of Gaps and End-to-End Correlations in Random Transverse-Field Ising Spin Chains, Phys. Rev. B 58, 9131 (1998).

[100] G. Kamienartz and H. W. J. Blöte, Universal Ratio of Magnetization Moments in Two-Dimensional Ising Models, J. Phys. A 26, 201 (1993).

[101] W. Selke, The Critical Binder Cumulant for Isotropic Ising Models on Square and Triangular Lattices, J. Stat. Mech. (2007) P04008.

[102] S. Yasuda and S. Todo, Monte Carlo Simulation with Aspect-Ratio Optimization: Anomalous Anisotropic Scaling in Dimerized Antiferromagnets, Phys. Rev. E 88, 061301(R) (2013).

[103] A. V. Chubukov, S. Sachdev, and J. Ye, Theory of TwoDimensional Quantum Heisenberg Antiferromagnets with a Nearly Critical Ground State, Phys. Rev. B 49, 11919 (1994).

[104] R. B. Griffiths, Nonanalytic Behavior Above the Critical Point in a Random Ising Ferromagnet, Phys. Rev. Lett. 23, 17 (1969).

[105] B. M. McCoy, Incompleteness of the Critical Exponent Description for Ferromagnetic Systems Containing Random Impurities, Phys. Rev. Lett. 23, 383 (1969).

[106] G. Grinstein, A. N. Berker, J. Chalupa, and M. Wortis, Exact Renormalization Group with Griffiths Singularities and Spin-Glass Behavior: The Random Ising Chain, Phys. Rev. Lett. 36, 1508 (1976).

[107] Y. Wang, W. Guo, and A. W. Sandvik, Anomalous Quantum Glass of Bosons in a Random Potential in Two Dimensions, Phys. Rev. Lett. 114, 105303 (2015).

[108] T. Roscilde and S. Haas, Mott Glass in Site-Diluted $S=1$ Antiferromagnets with Single-Ion Anisotropy, Phys. Rev. Lett. 99, 047205 (2007).

[109] N. Ma, D.-X. Yao, and A. W. Sandvik, Criticality and Mott Glass Phase in a Disordered Two-Dimensional Quantum Spin System, Phys. Rev. B 90, 104425 (2014).

[110] R. A. Hyman, K. Yang, R. N. Bhatt, and S. M. Girvin, Random Bonds and Topological Stability in Gapped Quantum Spin Chains, Phys. Rev. Lett. 76, 839 (1996).

[111] N. Laflorencie, D. Poilblanc, and A. W. Sandvik, Magnetic Ordering in a Doped Frustrated Spin-Peierls System, Phys. Rev. B 69, 212412 (2004).

[112] Another possible definition of the string density is to use $\rho=1$ for the covered sites, or one can just consider the locations of the unpaired spins (which carry the net spin) as defining the spinon density [98].

[113] In principle, the stability of the BRSFP to weak frustration can be studied in the RS phase by computing the scaling 
dimension of the operator used to define the frustrated terms on the square lattice (i.e., next-nearest-neighbor exchange interactions), which in practice can be done by analyzing the decay of the corresponding correlation function. Even without doing such a calculation, it seems most likely that the state we study here should not be unstable to weak frustration because the scaling dimension of an added interaction such as next-nearest-neighbor exchange is not dependent on the sign of the interactions. Since next-nearest-neighbor interactions with negative (ferromagnetic) sign are not frustrated, we do not see any reason for instability in this case, even when the sign is positive (frustrated interactions). Large-scale, low- $T$ QMC simulations with frustrated interactions explicitly included are essentially impossible due to the exponential increase in the severity of the sign problem (the small mean value of the \pm 1 sign factor of the sampled configurations) with increasing $L$ or $\beta$, even for weak frustration.

[114] Z. Ma et al., Spin-Glass Ground State in a TriangularLattice Compound $\mathrm{YbZnGaO}_{4}$, Phys. Rev. Lett. 120, 087201 (2018).

[115] R. K. K. Kaul, R. G. Melko, and A. W. Sandvik, Bridging Lattice-Scale Physics and Continuum Field Theory with Quantum Monte Carlo Simulations, Annu. Rev. Condens. Matter Phys. 4, 179 (2013).

[116] A. M. Goldsborough and R. A. Römer, Self-Assembling Tensor Networks and Holography in Disordered Spin Chains, Phys. Rev. B 89, 214203 (2014).

[117] Y.-P. Lin, Y.-J. Kao, P. Chen, and Y.-C. Lin, Griffiths Singularities in the Random Quantum Ising Antiferromagnet: A Tree Tensor Network Renormalization Group Study, Phys. Rev. B 96, 064427 (2017).

[118] J. C. Xavier, J. A. Hoyos, and E. Miranda, Adaptive Density Matrix Renormalization Group for Disordered Systems, arXiv:1809.04029 [Phys. Rev. B (to be published)].

[119] F. Alet, K. Damle, and S. Pujari, Sign-Problem-Free Monte Carlo Simulation of Certain Frustrated Quantum Magnets, Phys. Rev. Lett. 117, 197203 (2016).
[120] J. Stapmanns, P. Corboz, F. Mila, A. Honecker, B. Normand, and S. Wessel, Thermal Critical Points and Quantum Critical End Point in the Frustrated Bilayer Heisenberg Antiferromagnet, Phys. Rev. Lett. 121, 127201 (2018).

[121] S. Wessel, I. Niesen, J. Stapmanns, B. Normand, F. Mila, P. Corboz, and A. Honecker, Thermodynamic Properties of the Shastry-Sutherland Model from Quantum Monte Carlo Simulations, arXiv:1808.02043.

[122] A. Iaizzi, K. Damle, and A. W. Sandvik, Field-Driven Quantum Phase Transitions in $S=1 / 2$ Spin Chains, Phys. Rev. B 95, 174436 (2017).

[123] A. Iaizzi, K. Damle, and A. W. Sandvik, Metamagnetism and Zero-Scale-Factor Universality in the TwoDimensional J-Q model, Phys. Rev. B 98, 064405 (2018).

[124] H. Shao, Y. Q. Qin, S. Capponi, S. Chesi, Z. Y. Meng, and A. W. Sandvik, Nearly Deconfined Spinon Excitations in the Square-Lattice Spin-1/2 Heisenberg Antiferromagnet, Phys. Rev. X 7, 041072 (2017).

[125] Y.-R. Shu, M. Dupont, D.-X. Yao, S. Capponi, and A. W. Sandvik, Dynamical Properties of the $S=1 / 2$ Random Heisenberg Chain, Phys. Rev. B 97, 104424 (2018).

[126] K. Riedl, R. Valenti, and S. M. Winter, Critical Spin Liquid versus Valence Bond Glass in Triangular Lattice Organic $\kappa-(\mathrm{ET})_{2} \mathrm{Cu}_{2}(\mathrm{CN})_{3}$, arXiv:1808.03868.

[127] Preliminary results for the $J-Q_{2}$ model with random $J$ and $Q_{2}$ look very similar to those that we present here for the random $J-Q_{3}$ model. For example, in the system with bimodal random $Q_{2} \in\{0,2 Q\}$, we observe behaviors like in Fig. 19, with the susceptibility at $Q / J \approx$ 2.5 converging toward a $T$-independent form with increasing system size, while a slowly divergent $T \rightarrow 0$ behavior is seen for larger $Q$. These results support a universal $z=2$ at the AFM-RS transition and $z>2$ inside the RS phase. 